\title{
Reconstructing Gene Trees From Fitch's Xenology Relation
}

\author{
Manuela Geiß $^{1}$, John Anders ${ }^{1}$, Peter F. Stadler ${ }^{1,5,6,7}$, Nicolas Wieseke ${ }^{8}$, and Marc Hellmuth ${ }^{2,3}$ \\ ${ }^{1}$ Bioinformatics Group, Department of Computer Science; and Interdisciplinary Center of \\ Bioinformatics, University of Leipzig, , Härtelstraße 16-18, D-04107 Leipzig \\ ${ }^{2}$ Dpt. of Mathematics and Computer Science, University of Greifswald, Walther- Rathenau-Strasse 47, D-17487 Greifswald, \\ Germany, mhellmuth@mailbox.org \\ ${ }^{3}$ Saarland University, Center for Bioinformatics, Building E 2.1, P.O. Box 151150, D-66041 Saarbrücken, Germany \\ ${ }^{4}$ Department of Mathematics and Computer Science, University of Southern Denmark, Denmark \\ ${ }^{5}$ Max-Planck-Institute for Mathematics in the Sciences, , Inselstraße 22, D-04103 Leipzig \\ ${ }^{6}$ Inst. f. Theoretical Chemistry, University of Vienna, , Währingerstraße 17, A-1090 Wien, Austria \\ ${ }^{7}$ Santa Fe Institute, 1399 Hyde Park Rd., Santa Fe, USA \\ ${ }^{8}$ Parallel Computing and Complex Systems Group, Department of Computer Science, Leipzig University, Augustusplatz 10, 04109, \\ Leipzig, Germany
}

\begin{abstract}
Two genes are xenologs in the sense of Fitch if they are separated by at least one horizontal gene transfer event. Horizonal gene transfer is asymmetric in the sense that the transferred copy is distinguished from the one that remains within the ancestral lineage. Hence xenology is more precisely thought of as a nonsymmetric relation: $y$ is xenologous to $x$ if $y$ has been horizontally transferred at least once since it diverged from the least common ancestor of $x$ and $y$. We show that xenology relations are characterized by a small set of forbidden induced subgraphs on three vertices. Furthermore, each xenology relation can be derived from a unique least-resolved edge-labeled phylogenetic tree. We provide a linear-time algorithm for the recognition of xenology relations and for the construction of its least-resolved edge-labeled phylogenetic tree. The fact that being a xenology relation is a heritable graph property, finally has far-reaching consequences on approximation problems associated with xenology relations.
\end{abstract}

Keywords: Fitch Xenology; Phylogenetic Tree; Least-Resolved Tree; Informative Triple Sets; Di-Cograph; Heritable Graph Property; Forbidden Induced Subgraphs; Recognition Algorithm; Fixed Parameter Tractable

\section{Introduction}

The history of a gene family is defined by a phylogenetic tree of the genes (the gene tree), together with an event labeling of its inner vertices that identifies gene duplications, speciations events, and possibly horizontal gene transfer, as well as a mapping of the gene tree onto a species tree. The latter provides an implicit dating of the events that generated the gene phylogeny relative to the phylogeny of the species under consideration. The mathematical structure of gene family histories, i.e., the mutual relationships between gene trees, event labelings, species trees, and the corresponding reconciliation maps has only recently been explored in detail.

The concept of orthologs, that is, pairs of genes from different species that arose from a speciation event [14], play a key role in evolutionary biology. While functional similarity is not a defining feature of orthology, in general, orthologous genes from closely related species have a similar function. More strictly, one-to-one orthologs are in most cases functionally equivalent. Paralogs, that is pairs of genes that arose from duplication events, in contrast, often have related, but clearly distinct functions [30]. Orthologs, furthermore, tend to evolve in a clock-like fashion (at least as long as there are no additional duplications), which makes them the characters of choice in molecular phylogenetics [16].

The orthology relation on a set of genes forms a co-graph, whose associated co-tree is a not necessarily fully resolved event-labeled gene tree $[3,20]$. This result in particular implies that empirical estimates of the orthology relation, which are feasible in practise [2], provide direct information on the gene history. 
Empirically estimated orthology relationships in general violate the co-graph property, suggesting co-graph editing as a means to correct the initial estimate [11, 12, 31-34]. The event-labeled gene trees in turn constrain the possible species trees with which they can be reconciled [23, 26]. Given data on enough gene families, these constraints can be strong enough to completly specify also the species phylogeny [24].

Horizontal gene transfer is intimately related to the concept of xenology. A formal definition of xenology is less well established and by no means consistent in the biological literature. First we note that horizontal transfer is intrinsically a directional event, i.e., there is a clear distinction between the horizontally transferred "copy" and the "original" that continues to be vertically transferred. This fact can be annotated in the gene tree by associating a label to the edge that points from the horizontal transfer event to the next event in the history of the copy $[19,39]$. In $[22,38]$ this label was interpreted as a direction, leading to a notion of directed co-graphs [8], which turned out to be intimately related to so-called uniformly non-prime 2-structures, see [22].

The most commonly used definition in the biological literature, introduced by Walter M. Fitch in 2000, calls a pair of genes xenologs if the history since their common ancestor involves horizontal transfer of at least one of them $[15,29]$. Preserving the directionality of horizontal transfer, we capture this concept with the help of a non-symmetric xenology relation $X$ on a set of genes such that $(x, y) \in X$ whenever there is at least one directed horizontal transfer event during the evolution from the last common ancestor of $x$ and $y$ towards $y$.

While best match heuristics have been very successful as approximations of the orthology relation [2, 37], no comparable approach to extract the xenology relation directly from (dis)similarity data has been devised to-date. We suspect that this is at least one reason why the binary xenology relation has attracted very little attention so far. Nevertheless, there are several methods to detect xenologs in a genome that use sequence features rather then phylogenetic reconstructions, see e.g. [41, 42]. In this contribution we focus on the mathematical properties of the xenology relation $X$. In particular, we will be concerned with two related questions: (1) How much information on the gene tree $T$ and the location of the horizontal transfer events within $T$ is contained in the xenology relation? (2) Is it possible to extract the topological information and labeling information from $X$ efficiently?

We show here that valid xenology relations correspond to a heritable family of di-graphs, which we call the Fitch graphs. These are characterized by a small set of forbidden subgraphs on three vertices and thus can be recognized in cubic time. Fitch graphs form a subclass of di-cographs, which have recently been associated with an alternative concept of xenology [22]. Each Fitch graph is explained by a unique leastresolved edge-labeled phylogenetic tree. This tree is displayed by the full evolutionary scenario. It therefore provides a least partial information on the gene tree and the placement of the horizontal transfer events. We will show, furthermore, that this tree as well as corresponding the edge-labeling can be constructed from $X$ in polynomial time. Utilizing features of heritable graph properties we derive a linear-time recognition algorithm, as well as NP-completeness and fixed-parameter tractable results for the respective graph modification problems. We take these results as motivation for future work towards methods to estimate the xenology relation from sequence (dis)similarity data.

\section{Preliminaries: Rooted Trees, Phylogenetic Trees and Rooted Triples}

A rooted tree $T=(V, E)$ with leaf set $L \subseteq V$ (or $L(T)$ in case of ambiguity) and inner vertices $V^{0}=V \backslash L$ is an acyclic connected graph containing one distinguished inner vertex $\rho_{T} \in V^{0}$ that is called the root of T. The degree of a vertex $v \in V$ is denoted by $\operatorname{deg}(v)$. The root $\rho_{T}$ is regarded as an inner vertex, i.e. $\rho_{T} \notin L$, even if $\operatorname{deg}\left(\rho_{T}\right)=1$. A rooted tree $T=(V, E)$ on $L$ is phylogenetic if its root has at least $\operatorname{deg}\left(\rho_{T}\right) \geq 2$ and every other inner vertex $v \in V^{0} \backslash\left\{\rho_{T}\right\}$ has $\operatorname{deg}(v) \geq 3$. If the degree of each vertex $v \in V^{0} \backslash\left\{\rho_{T}\right\}$ is exactly three and $\operatorname{deg}\left(\rho_{T}\right)=2$, then the phylogenetic tree is called binary. In this contribution, we will consider rooted trees together with an edge-labeling $\lambda: E \rightarrow\{0,1\}$ and write $(T, \lambda)$.

We call $u \in V$ an ancestor of $v \in V, u \succeq_{T} v$, and $v$ a descendant of $u, v \preceq_{T} u$, if $u$ lies on the unique path from $\rho_{T}$ to $v$. We write $v \prec_{T} u\left(u \succ_{T} v\right)$ for $v \preceq_{T} u\left(u \succeq_{T} v\right)$ and $u \neq v$. If $v \preceq_{T} u$ or $u \succeq_{T} v$, then $u$ and $v$ are comparable, and incomparable otherwise. It will be convenient to use a notation for edges $e$ that implies which of the vertex in $e$ is closer to the root, that is, we always write $(u, v) \in E$ to indicate that $u \succ_{T} v$. In the latter case, vertex $u$ is called parent of $v$, denoted by $\operatorname{par}(v)$. Similarly, we define the children of $u$ as $\operatorname{child}(u):=\{v \in V \mid(u, v) \in E\}$. We denote two leaves $v, w \in L$ as siblings if $v, w \in \operatorname{child}(u)$. Edges that are incident to a leaf are called outer edges. Conversely, inner edges do only contain inner vertices.

For a non-empty subset $L^{\prime} \subseteq L$ of leaves, the least common ancestor of $L^{\prime}$, denoted as lca $\left(L^{\prime}\right)$, is the unique $\preceq_{T}$-minimal vertex of $T$ that is an ancestor of every vertex in $L^{\prime}$. We will make use of the simplified 
notation $\operatorname{lca}_{T}(x, y, z):=\operatorname{lca}_{T}(\{x, y, z\})$ for $L^{\prime}=\{x, y, z\}$ and we will omit the explicit reference to $T$ whenever it is clear which tree is considered. Analogously, we often write $\operatorname{deg}(v)$ instead of $\operatorname{deg}_{T}(v)$ for the degree of some vertex $v$.

A simple contraction of an edge $e=(x, y)$ in a tree $T$ refers to the removal of $e$ and identification of $x$ and $y$. The tree $T\left(L^{\prime}\right)$ with root $\operatorname{lca}_{T}\left(L^{\prime}\right)$ has leaf set $L^{\prime}$ and consists of all paths in $T$ that connect the leaves in $L^{\prime}$. We say that a rooted tree $T$ on $L$ displays a root tree $T^{\prime}$ on $L^{\prime}$, in symbols $T^{\prime} \leq T$, if $T^{\prime}$ can be obtained from $T\left(L^{\prime}\right)$ by a sequence of simple edge contractions. We write $T^{\prime}<T$ if $T^{\prime} \leq T$ and $T^{\prime} \neq T$. The restriction $T \mid L^{\prime}$ of $T$ to $L^{\prime}$ is the rooted tree obtained from $T\left(L^{\prime}\right)$ by suppressing all vertices of degree 2 with the exception of the root $\rho_{T}$ if $\rho_{T} \in V\left(T\left(L^{\prime}\right)\right)$. By construction, $T \mid L^{\prime}$ is a phylogenetic tree. The suppression of vertices of degree 2 can be achieved by simple contraction of one of the adjacent edges. Moreover, $T \mid L^{\prime} \leq T$, i.e., $T$ displays the restrictions $T \mid L^{\prime}$ to all subsets $L^{\prime} \subseteq L$. Note that $T \mid L=T$ if and only if $T$ is phylogenetic; otherwise $T \mid L<T$.

For every vertex $v \in V(T)$ we denote by $C(v)$ the subset of $L$ such that $\forall x \in L$ it holds that $x \in C(v)$ if and only if $x \preceq_{T} v$. Moreover, we define $\mathcal{C}(T):=\{C(v) \mid v \in V(T)\}$. A rooted tree is phylogenetic if and only if $C(u)=C(v)$ implies $u=v$ for all $u, v \in E(T)$. We say that a rooted tree $T^{\prime}$ on $L$ refines a rooted tree $T$ on $L$, if $T^{\prime}$ displays $T$. In particular, a phylogenetic tree $T^{\prime}$ on $L$ refines a rooted tree $T$ if and only if $\mathrm{C}(T) \subseteq \mathcal{C}\left(T^{\prime}\right)$. In particular, the tree $T(v)$ rooted at a vertex $v$ of $T$ is the tree $T(C(v))$.

Rooted triples are binary rooted phylogenetic trees on three leaves. We write $a b \mid c$ for the rooted triple with leaves $a, b$ and $c$, if the path from its root to $c$ does not intersect the path from $a$ to $b$. The definition of "display" implies that a triple $a b \mid c$ with $a, b, c \in L$ is displayed by a rooted tree $T$ if lca $(a, b) \prec_{T} \operatorname{lca}(a, b, c)$.

The set of all triples that are displayed by $T$ is denoted by $r(T)$. For a set $R$ of rooted triples we define $R_{x} \subseteq R$ as the set of triples in $R$ that contain the leaf $x$. A set of rooted triples $R$ is called consistent if there exists a phylogenetic tree $T$ on $L_{R}:=\bigcup_{a b \mid c \in R}\{a, b, c\}$ that displays $R$, i.e., $R \subseteq r(T)$. In particular, a tree can display at most one triple on any set of three leaves. Thus a triple set $R$ is inconsistent whenever $a b|c, a c| b \in R$. However, triple sets can be inconsistent even if they do not contain two triples on the same three leaves.

Rooted triples are widely used in the context of supertree reconstruction because every phylogenetic tree $T$ is identified by its triple set $r(T)$, and $r(T) \subseteq r\left(T^{\prime}\right)$ if and only if $T^{\prime}$ displays $T$ [43]. As a consequence, supertree reconstruction can be phrased in terms of triples. As shown in [1] there is a polynomial-time algorithm, usually referred to as BUILD [43,44], that takes a set $R$ of triples as input and either returns a particular phylogenetic tree Aho $(R)$ that displays $R$, or recognizes $R$ as inconsistent.

The requirement that a set $R$ of triples is consistent, and thus, that there is a tree displaying all triples, makes it possible to infer new triples from the trees that display $R$ and to define a closure operation for $R$ $[4,5,17,21]$. Let $\langle R\rangle$ be the set of all rooted trees with leaf set $L_{R}$ that display $R$. The closure of a consistent set of rooted triples $R$ is defined as

$$
\operatorname{cl}(R)=\bigcap_{T \in\langle R\rangle} r(T) .
$$

Hence, a triple $r$ is contained in the closure $\operatorname{cl}(R)$ if all trees that display $R$ also display $r$. This operation satisfies the usual three properties of a closure operator [5], namely: (i) expansiveness, $R \subseteq \operatorname{cl}(R)$; (ii) isotony, $R^{\prime} \subseteq R$ implies that $\operatorname{cl}\left(R^{\prime}\right) \subseteq \operatorname{cl}(R)$; and (iii) idempotency, $\operatorname{cl}(\operatorname{cl}(R))=\operatorname{cl}(R)$. Since $T \in\langle r(T)\rangle$, it is easy to see that $\operatorname{cl}(r(T))=r(T)$ and thus, $r(T)$ is always closed.

A set of rooted triples $R$ identifies a tree $T$ with leaf set $L_{R}$ if $R$ is displayed by $T$ and every other tree $T^{\prime}$ that displays $R$ is a refinement of $T$. A rooted triple $a b \mid c \in r(T)$ distinguishes an edge $(u, v)$ in $T$ iff $a, b$, and $c$ are descendants of $u, v$ is an ancestor of $a$ and $b$ but not of $c$, and there is no descendant $v^{\prime}$ of $v$ for which $a$ and $b$ are both descendants. In other words, $a b \mid c \in r(T)$ distinguishes the edge $(u, v)$ if lca $(a, b)=v$ and $\operatorname{lca}(a, b, c)=u$.

We will make use of two results from [17] that are closely related to the BUILD algorithm.

Lemma 1. Let $T$ be a phylogenetic tree and let $R$ be a set of rooted triples. Then, $R$ identifies $T$ if and only if $\operatorname{cl}(R)=r(T)$. Moreover, if $R$ identifies $T$, then $\operatorname{Aho}(R)=T$.

\section{The (Fitch-)Xenology Relation}

In this contribution we are specifically interested in phylogenetic trees $T=(V, E)$ with leaf set $L=L(T)$ that are endowed with edge labels $\lambda: E \rightarrow\{0,1\}$ such that

$$
\lambda(e)= \begin{cases}1 & \text { if } e \text { is a horizontal transfer-edge } \\ 0 & \text { otherwise }\end{cases}
$$

For simplicity we will speak of 0 -edges and 1-edges in $T$ depending on their labeling. 
a)

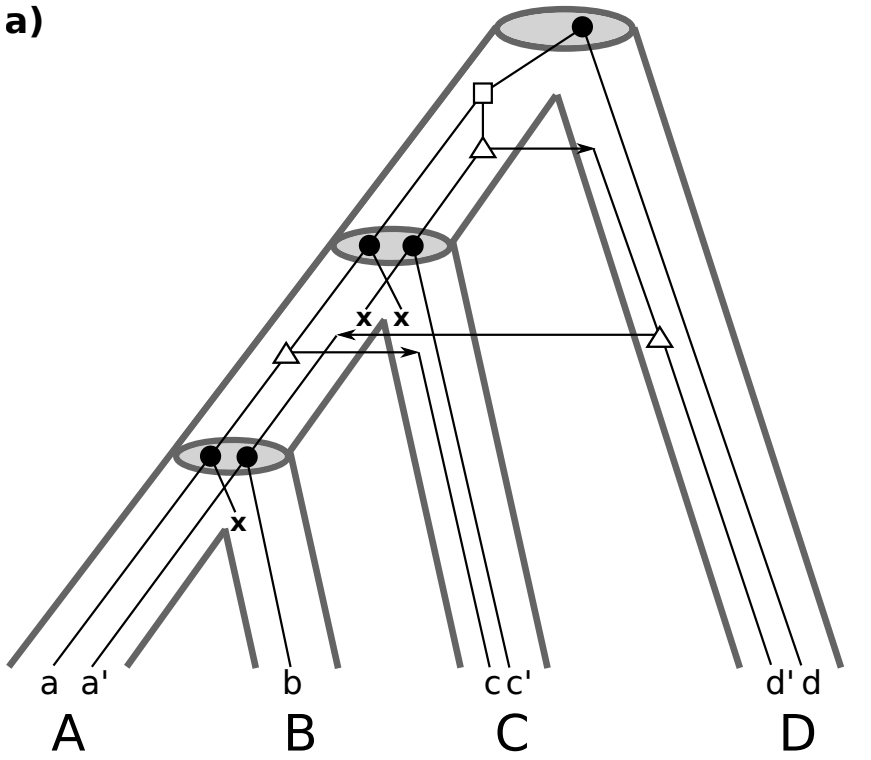

b)

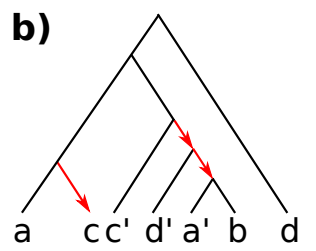

c)

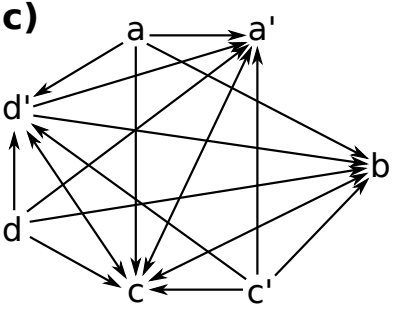

Figure 1: a) Event-labeled gene tree embedded in the (tube-like) species tree. The leaf set of the gene tree are the genes $a, a^{\prime}, b, c, c^{\prime}, d$, and $d^{\prime}$ in the genomes of the four species $A, B, C$ and $D$. The gene tree contains speciations $(\bullet)$, duplications $(\square)$, HGT events $(\triangle)$ and gene losses $(\times)$. b) Removal of all gene losses, suppression of all resulting degree two vertices and ignoring the types of the events on the vertices yields an edge-labeled tree in which the transfer edges labeled by 1 (red arrow) and all other edges by 0 (black edges). Panel c) shows the Fitch graph explained by the edge-labeled tree of Panel b).

Definition 1. Given an edge-labeled phylogenetic tree $(T, \lambda)$ we set $(x, y) \in X_{(T, \lambda)}$ for $x, y \in L$ whenever there is at least one directed horizontal transfer event between $y$ and the last common ancestor of $x$ and $y$, i.e., if the uniquely defined path from $\operatorname{lca}_{T}(x, y)$ to y contains at least one 1-edge. We write $[x, y] \in X_{(T, \lambda)}$ if $(x, y)$ and $(y, x) \in X_{(T, \lambda)}$ and $x \mid y$ if $(x, y)$ and $(y, x) \notin X_{(T, \lambda)}$.

By construction $X_{(T, \lambda)}$ is irreflexive; hence it can be regarded as a simple directed graph. In the following, we therefore will interchangeably speak of $X_{(T, \lambda)}$ as graph or relation and use the standard graph terminology such as "induced subgraph in $X_{(T, \lambda)}$ ". It is easy to check that $X_{(T, \lambda)}$ is in general neither symmetric nor antisymmetric. The relation $X_{(T, \lambda)}$ formalizes Fitch's concept of xenology [15].

We say that an edge-labeled phylogenetic tree $(T, \lambda)$ explains a given irreflexive relation $X$ whenever $X=X_{(T, \lambda)}$. To be more explicit, $(T, \lambda)$ explains $X$ if there is a 1-edge on the path from lca $(x, y)$ to $y$ if and only if $(x, y) \in X$. By construction, $X$ must be defined on $L(T)$. We call a relation $X$ valid if there is an edge-labeled tree that explains $X$. An example of a gene tree with the corresponding Fitch relation $X$ and an edge-labeled tree that explains $X$, can be found in Fig. 1 .

The notion of a tree $T^{\prime}$ being displayed by a tree $T$ can be generalized to edge-labeled trees: We say that $\left(T^{\prime}, \lambda^{\prime}\right)$ is displayed by $(T, \lambda)$ if $T^{\prime}$ is displayed by $T$ in the usual sense and an edge $e^{\prime} \in E\left(T^{\prime}\right)$ has label $\lambda^{\prime}\left(e^{\prime}\right)=1$ if and only if the path in $T$ that corresponds to $e^{\prime}$ contains at least one 1-edge.

Lemma 2. Let $\left(T^{\prime}, \lambda^{\prime}\right)$ be a tree with leaf set $L^{\prime}=L\left(T^{\prime}\right)$ that is displayed by $(T, \lambda)$. Then $X_{\left(T^{\prime}, \lambda^{\prime}\right)}$ is the subgraph of $X_{(T, \lambda)}$ induced by $L^{\prime}$.

Proof. Consider two distinct leaves $x, y \in L^{\prime}$. By construction of $\left(T^{\prime}, \lambda^{\prime}\right)$ there is a 1-edge on the path from $\operatorname{lca}_{T^{\prime}}(x, y)$ to the leaf $y$ in $\left(T^{\prime}, \lambda^{\prime}\right)$ if and only if the corresponding path in $(T, \lambda)$ containes a 1 -edge and thus $(x, y) \in \mathcal{X}_{\left(T^{\prime}, \lambda^{\prime}\right)}$ iff $(x, y) \in X_{(T, \lambda)}$.

The enumeration of all edge-labeled trees on two vertices shows that all four possible digraphs on two vertices are valid. For three vertices, however, there are valid and invalid digraphs. These are summarized in Figure 2: up to isomorphism there are eight valid $A_{1}-A_{8}$ and eight invalid $F_{1}-F_{8}$ digraphs. We will refer to them as valid and invalid triangles. We denote subgraphs of $X$ that are induced by the vertices $x_{1}, \ldots, x_{k}$ by $X\left[x_{1}, \ldots, x_{k}\right]$. In particular, triangles in $X$ are denoted by $X[a, b, c]$, where $a, b, c \in L$ are three distinct vertices.

Definition 2. An irreflexive binary relation $X$ on $L$ is a Fitch relation if all its triangles are valid. Its graph representation is called $a$ Fitch graph. 


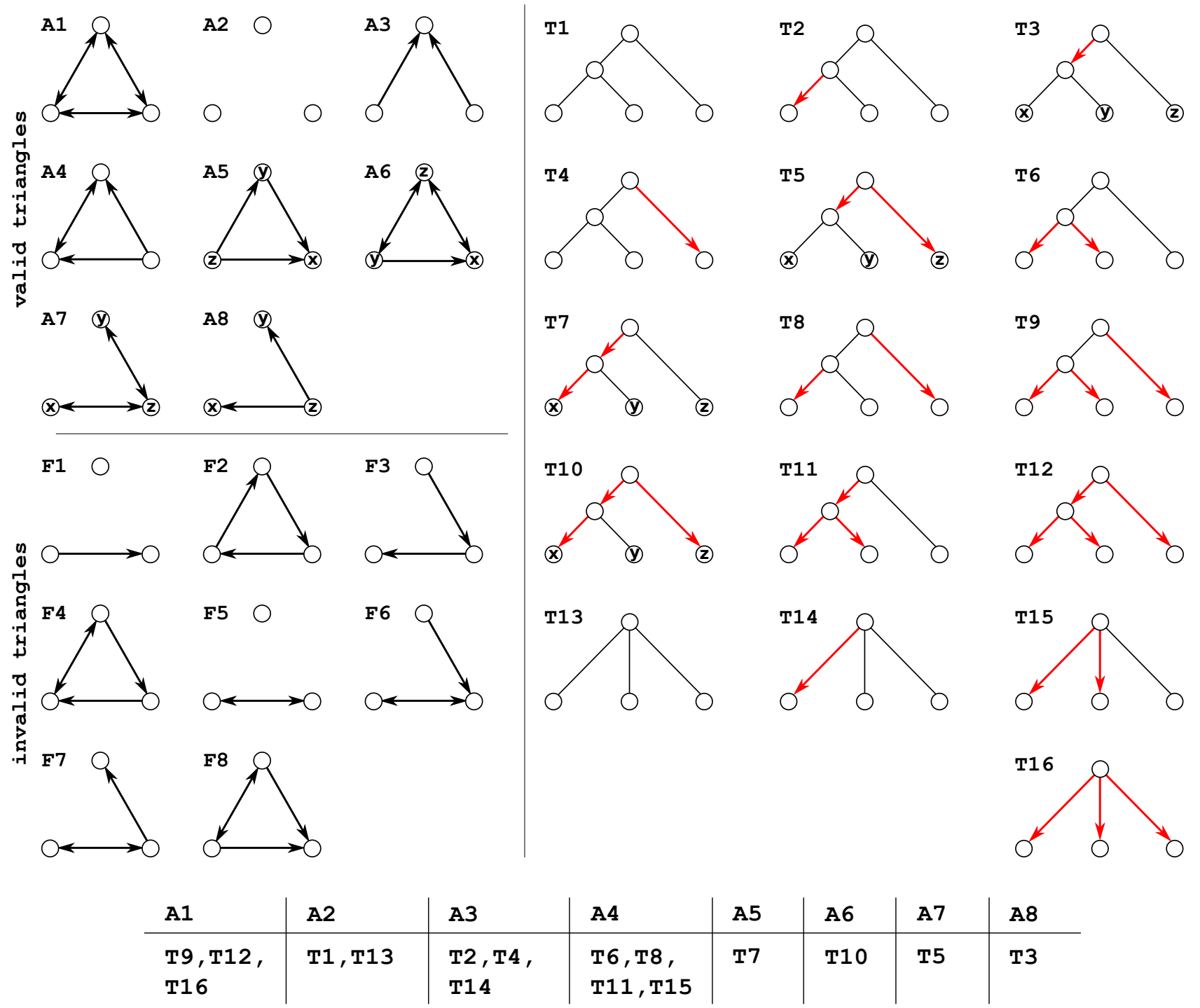

Figure 2: Upper Left: Shown is the graph representation for all possible relations $X \subseteq L \times L$ with $|L|=3$. The relations are grouped into valid $\left(A_{1}-A_{8}\right)$ and non-valid $\left(F_{1}-F_{8}\right)$.

Upper Right: All possible (up to isomorphism) subtrees on three leaves of a tree $(T, \lambda)$ are shown. Edges can be understood as paths, whereby red (resp. black) edges indicate that there is (resp., is not) a 1-edge on the particular path.

Lower Part: The table shows which tree explains which relation. In particular, there is no tree that would explain one of the graphs $F_{1}$ to $F_{8}$.

A graph $G$ is a di-cograph if and only if it does not contain one of the digraphs shown in Fig.3 as an induced subgraph [8]. Since each of these graphs contains one of the forbidden triangles, every Fitch graph is also a di-cograph. On the other hand, a di-cograph that does not contain $F_{1}, F_{5}$, or $F_{8}$ as an induced subgraph is a Fitch graph. As an immediate consequence of its characterization in terms of forbidden induced subgraphs, Fitch graphs are a heritable family, i.e., every induced subgraph of a Fitch graph is again a Fitch graph. We summarize these observations for later reference as

Lemma 3. The Fitch graphs are a heritable subfamily of the di-cographs.

A closer inspection shows that four of the eight valid triangles, $A_{1}-A_{4}$ can be explained by multiple trees, including one of the non-binary trees $T_{13}$ to $T_{16}$. In contrast, each of the triangles $A_{5}-A_{8}$ with a given labeling of its three leaves is explained by a unique edge-labeled binary tree, i.e., a specific labeled triple.

Definition 3. An edge-labeled triple $a b \mid c$ is informative if it explains a labeled triangle isomorphic to one of $A_{5}, A_{6}, A_{7}$ or $A_{8}$.

Thus, if $X$ contains a triangle of the form $A_{5}, A_{6}, A_{7}$ or $A_{8}$ as an induced subgraph, then any tree explaining $X$ must display the corresponding informative triple. Any valid relation $X$ can therefore be 

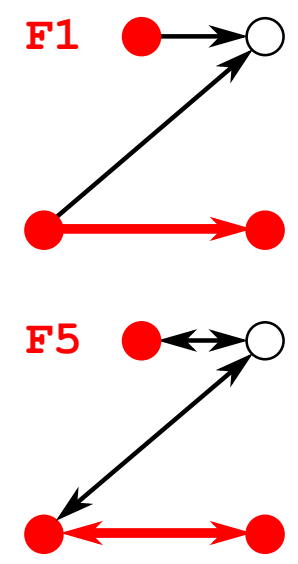
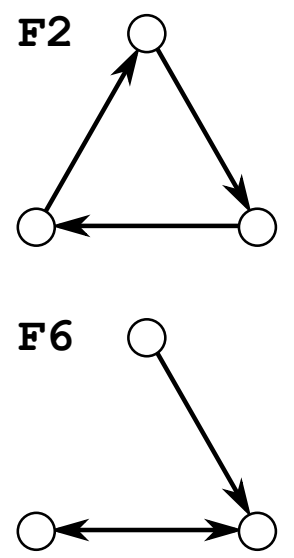

F3
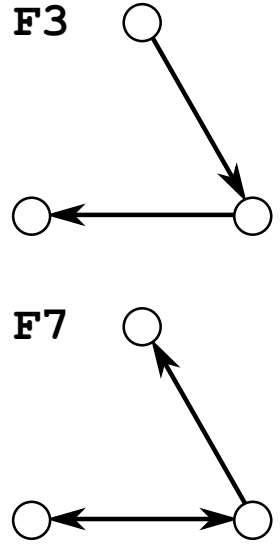
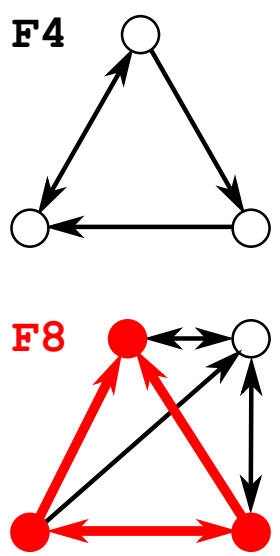

Figure 3: The eight digraphs are the forbidden induced subgraphs that characterize di-cographs [8, 13]. The five digraphs on three vertices correspond to five of the eight forbidden triangles. Each digraph on four vertices contains one of the remaining forbidden triangles (highlighted by bold-red edges and vertices).

associated with a uniquely defined set $r_{I}(X)$ of informative triples that it displays: $r \in r_{I}(X)$ if and only if $r$ is the unique edge-labeled triple explaining an induced triangle isomorphic to $A_{5}, A_{6}, A_{7}$ or $A_{8}$. For later reference we summarize this fact as

Lemma 4. If $(T, \lambda)$ explains $X$, then all triples in $r_{I}(X)$ must be displayed by $(T, \lambda)$.

\section{Least-Resolved Edge-Labeled Phylogenetic Trees}

In general, there may be more than one rooted (phylogenetic) tree that explains a given relation $X$. In particular, if $\mathcal{X}$ is explained by a non-binary tree $(T, \lambda)$, then there is always a binary tree $\left(T^{\prime}, \lambda^{\prime}\right)$ that refines $T$ and explains the same relation $X$ by setting $\lambda^{\prime}(e)=\lambda(e)$ for all edges $e$ that are also in $T$ and by choosing the label $\lambda^{\prime}(e)=0$ for all edges $e$ that are not contained in $T$. In this section, we will show that whenever a relation $X$ is explained by an edge-labeled tree $(T, \lambda)$, then there exists a unique "smallest" tree with this property, which we will call the least-resolved tree. These least resolved trees will play a key role for obtaining a characterization of Fitch relations in the following.

Definition 4. Let $(T=(V, E), \lambda)$ be an edge-labeled phylogenetic tree and let $e=(x, y) \in E$. The phylogenetic tree $\left(T_{e}, \lambda_{e}\right)$, referred to as the (extended) contraction of $e$ in $(T, \lambda)$, is obtained by the following procedure:

First contract the edge $e$ in $T$ and keep the edge-labels of all non-contracted edges. If $e$ is an inner edge, the resulting tree is again a phylogenetic tree and we are done. The contraction of an outer edge $e=(u, v)$, however, leads to ( $i$ ) the loss of a leaf $v$ and (ii) a decrease in the degree of the parental vertex $u$. The latter may violate the degree conditions required for a phylogenetic tree. If $u$ is the root of $T$ that has degree 1 in $T_{e}$, we delete $u$ and its incident edge, and declare the unique remaining child of $u$ as the root of $T_{e}$. Thus, $T_{e}$ is obtained by an additional simple contraction of the edge $\left(\rho_{T_{e}}, \operatorname{child}\left(\rho_{T}\right)\right)$. Otherwise, if $u$ is an inner vertex that has degree 2 after the contraction of $e$, we apply an additional simple contraction of the edge $(u, \operatorname{child}(u))$ and set $\lambda(\operatorname{par}(u), u)=1$ if $\lambda(u, \operatorname{child}(u))=1$. Equivalently, the path from the parent $w$ of $u$ to the unique remaining child $w^{\prime}$ of $u$ is replaced by a single edge $\left(w, w^{\prime}\right)$. This edge is a 1-edge if and only if at least one of the edges wu and $u w^{\prime}$ in the initial tree was a 1-edge.

Definition 5. An edge-labeled phylogenetic tree $(T=(V, E), \lambda)$ is least-resolved (w.r.t. $\left.X_{(T, \lambda)}\right)$ if none of the edge-contracted trees $\left(T_{e}, \lambda_{e}\right), e \in E$, explains $X_{(T, \lambda)}$.

It is easy to see that $\left(T_{e}, \lambda_{e}\right)$ is, by construction, always obtained by a sequence of simple edge contractions and thus, $\left(T_{e}, \lambda_{e}\right)$ is displayed by $(T, \lambda)$.

Lemma 5. Let $(T, \lambda)$ be an edge-labeled phylogenetic tree. If e is an inner 0-edge in $(T, \lambda)$, then $X_{\left(T_{e}, \lambda_{e}\right)}=$ $x_{(T, \lambda)}$. If e is an inner 1-edge, then $x_{\left(T_{e}, \lambda_{e}\right)} \subseteq X_{(T, \lambda)}$.

Proof. The contraction of the inner 0-edge $e=(u, v)$ does not change the number of 1-edges along the paths connecting any two leaves. It affects the least common ancestor of $x$ and $y$, if $\operatorname{lca}_{T}(x, y)=u$ or $\operatorname{lca}_{T}(x, y)=v$. In either case, however, the number of 1-edges between the lca $T(x, y)$ and the leaves $x$ and $y$ remains unchanged. Hence, the relation $X_{(T, \lambda)}$ is not affected by the contraction. 
The contraction of a 1-edge $e$ reduces the number of 1-edges along the path between all pairs of leaves whose connecting path in $T$ contain $e$. Thus, if $(x, y) \in \mathcal{X}_{\left(T_{e}, \lambda_{e}\right)}$ then the path connecting $x$ and $y$ in $T$ contains also at least one 1-edge, and hence $(x, y) \in X_{(T, \lambda)}$

Note that edge contractions therefore always imply $X_{\left(T_{e}, \lambda_{e}\right)} \subseteq X_{(T, \lambda)}$. There may be edges in a tree whose labeling does not affect the relation, i.e., they can be labeled either 0 or 1 . The latter observation gives rise to the following definition.

Definition 6. An edge $e$ in a tree $(T, \lambda)$ is irrelevant if $\left(T, \lambda^{\prime}\right)$ with $\lambda^{\prime}(e) \neq \lambda(e)$ and $\lambda^{\prime}(f)=\lambda(f)$ for all $f \neq e$ still explains $X_{(T, \lambda)}$.

Edges that are not irrelevant are called relevant. As an example consider the two trees $T_{9}$ and $T_{12}$ in Figure 2. Both explain the valid triangle $A_{1}$. The inner edge of $T_{9}$ and $T_{12}$ is a 0 -edge and 1-edge, respectively. Thus, this edge is irrelevant. The tree $T_{16}$, which is obtained from both $T_{9}$ and $T_{12}$ by contracting the irrelevant edge, still explains $A_{1}$. For later reference, we provide a simple characterization of irrelevant edges.

Lemma 6. An edge $e=(u, v)$ is irrelevant in a phylogenetic tree $(T, \lambda)$ if and only if $e$ is an inner edge and every path from $v$ to each leaf in the subtree rooted at $v$ contains a 1-edge.

Proof. Any inner edge $e$ that satisfies the condition of the lemma is irrelevant because every path $u$ to a leaf contains a 1-edge irrespective of the label of $(u, v)$.

Conversely, assume first that $e=(u, v)$ is an outer edge. Hence, changing the label of $e$ would immediately change the relation between $v$ and any leaf $w$ located in a subtree rooted at a sibling of $v$. Since at least one such leaf $w$ exists in a phylogenetic tree, $e$ is relevant. Now suppose that $e=(u, v)$ is an inner edge and that there is a leaf $w$ below $v$ such that the path from $v$ to $w$ comprises only 0-edges. Let $x$ be a leaf such that lca $(w, x)=u$. Since $T$ is a phylogenetic tree, such a leaf always exists. Then $(x, w) \in X$ if and only if $\lambda(e)=1$, i.e., the inner edge $e$ is relevant.

A crucial consequence of Lemma 6 is that every outer edge is relevant. Furthermore, since an irrelevant edge can be relabeled as a 0 -edge without affecting $X_{(T, \lambda)}$, Lemma 5 implies that irrelevant edges can be contracted without changing $X_{(T, \lambda)}$. These observations naturally pose the question how edge-labeled trees are structured that cannot be contracted further without affecting $X_{(T, \lambda)}$.

Lemma 7. Let $(T, \lambda)$ be an edge-labeled phylogenetic tree explaining $X$. Then, the tree $\left(T_{e}, \lambda_{e}\right)$ obtained by contracting the edge explains $X$ if and only if e is irrelevant or $e$ is an inner 0-edge.

Proof. The discussion above already shows that irrelevant edges as well as 0-edges can be contracted without affecting $X$. We show that $X_{\left(T_{e}, \lambda_{e}\right)} \neq X_{(T, \lambda)}$ whenever $e$ is an outer edge or a relevant inner 1-edge. First we assume that $e$ is an outer edge. Clearly, if $v$ is a leaf, then contracting $e=(u, v)$ would change $v$ to an inner vertex in $\left(T_{e}, \lambda_{e}\right)$. Thus, $L(T) \neq L\left(T^{\prime}\right)$ and therefore, $\left(T_{e}, \lambda_{e}\right)$ does not explain $X$. Now, let $e$ be a relevant inner 1-edge. Then, there is a leaf $x$ in the subtree rooted at $v$ such that $\operatorname{Path}(v, x)$ consists only of 0-edges (cf. Lemma 6). Since $(T, \lambda)$ is phylogenetic, there exists a leaf $y \in L(T)$ such that $\operatorname{lca}_{T}(x, y)=u$. Moreover, as $\lambda(u, v)=1$, we have $(y, x) \in X$. Contracting $e$ makes the vertex $u^{*}$, obtained by identifying $u$ and $v$, the least common ancestor of $x$ and $y$, i.e., $\operatorname{lca}_{T_{e}}(x, y)=u^{*}$. The path from $u^{*}$ to $x$ now contains only 0-edges, i.e., $(y, x) \notin X_{\left(T_{e}, \lambda_{e}\right)}$. Thus, relevant 1 -edges of $(T, \lambda)$ cannot be contracted without affecting $x$.

The following result shows that relevant edges in a tree $(T, \lambda)$ remain relevant in any of its edgecontracted versions $\left(T_{e}, \lambda_{e}\right)$, where $e$ is an inner 0-edge or an irrelevant edge.

Lemma 8. Let $(T, \lambda)$ be an edge-labeled phylogenetic tree explaining $X$, the edge e be an inner 0-edge or an irrelevant 1-edge in $(T, \lambda)$ and $\left(T_{e}, \lambda_{e}\right)$ be the tree obtained from $(T, \lambda)$ by contracting the edge $e$. Then, the edge $f \neq e$ is relevant in $\left(T_{e}, \lambda_{e}\right)$ if and only if $f$ is relevant in $(T, \lambda)$.

Proof. As a consequence of Lemma 7, $\left(T_{e}, \lambda_{e}\right)$ still explains $\mathcal{X}$. Lemma 6 implies that the edge $f=(u, v)$ is irrelevant in $\left(T_{e}, \lambda_{e}\right)$ if and only if $f$ is an inner edge and all paths from $v$ to leaves below $v$ contain a 1-edge. If $e$ is not located below $f$, then the contraction of $e$ does not affect this condition and thus, $f$ is irrelevant in $\left(T_{e}, \lambda_{e}\right)$ if and only if it is irrelevant in $(T, \lambda)$.

Now suppose $e$ is located below $f$. If $e$ was a 0-edge, the number of 1-edges along the paths from $v$ to the leaves does not change upon edge contraction, and thus $f$ is irrelevant in $\left(T_{e}, \lambda_{e}\right)$ if and only if it is irrelevant in $(T, \lambda)$. Finally, suppose $e=\left(u^{\prime}, v^{\prime}\right)$ was an irrelevant 1-edge. Thus, we can set $\lambda(e)=0$ in $(T, \lambda)$ without changing the relation $X$. Now we can repeat the latter arguments to conclude that $f$ is irrelevant in $\left(T_{e}, \lambda_{e}\right)$ if and only if it is irrelevant in $(T, \lambda)$. 
The following result shows that the order of the contraction of inner 0-edges or irrelevant 1-edges does not affect the resulting relation.

Lemma 9. Let $(T, \lambda)$ be an edge-labeled phylogenetic tree and let $e$ and $f$ be two edges in $E(T)$ such that $(T, \lambda),\left(T_{e}, \lambda_{e}\right)$ and $\left(T_{f}, \lambda_{f}\right)$ explain the same relation $X$. Then, $\left(\left(T_{e}\right)_{f},\left(\lambda_{e}\right)_{f}\right)$ obtained from $\left(T_{e}, \lambda_{e}\right)$ by contracting the edge $f$, also explains $X$.

Proof. By Lemma 7, an edge can be contracted without affecting $X$ if and only it is an inner 0-edge or an irrelevant 1-edge. The labeling of $f$ is not affected by contraction of $e$ and vice versa. Lemma 8 furthermore shows that the (ir)relevance of an edge $f \neq e$ is conserved by the contraction of 0 -edges and irrelevant 1-edges. Therefore $e$ and $f$ can be contracted in arbitrary order and preserve $X$ in each contraction step.

We will now apply the results developed so far to least-resolved trees. First, we show that the order of edge contractions does not affect the resulting least-resolved tree.

Lemma 10. Let $(T, \lambda)$ be a least-resolved tree w.r.t. $X=X_{(T, \lambda)}$. Then, there is no sequence of edge contractions $e_{1} e_{2} \ldots e_{\ell}$ such that the resulting contracted tree $T_{e_{1} e_{2} \ldots e_{\ell}}$ explains $X_{(T, \lambda)}$.

Proof. Let $(T, \lambda)$ be a least-resolved tree, i.e., none of the edge-contracted trees $\left(T_{e}, \lambda_{e}\right), e \in E$, explains $X_{(T, \lambda)}$. Lemma 5 and 7 imply that any edge $e \in E$ must be either an outer edge or a relevant 1-edge. Clearly, if any edge of the sequence $e_{1} e_{2} \ldots e_{\ell}$ is an outer edge, then the statement is trivially satisfied.

Hence, assume that all edges $e_{1} e_{2} \ldots e_{\ell}$ are inner edges and therefore, relevant 1-edges in $(T, \lambda)$. Lemma 5 implies that for $X$ to change, there must be at least one pair of leaves $x, y$ such that $(x, y) \in X_{(T, \lambda)}$ and $(x, y) \notin X_{\left(T_{e}, \lambda_{e}\right)}$, i.e., there is no 1-edge along $\operatorname{Path}(\operatorname{lca}(x, y), y)$ in $T_{e}$, and $e$ was the only 1-edge along $\operatorname{Path}(\operatorname{lca}(x, y), y)$ in $T$. By Lemma 5, $(x, y) \notin X^{\prime}$ for the relation explained by any tree that is obtained from edge contractions of $\left(T_{e}, \lambda_{e}\right)$, i.e., there is no sequence of edge contractions that leads to a tree $\left(T^{\prime}, \lambda^{\prime}\right)$ such that $\chi_{\left(T^{\prime}, \lambda^{\prime}\right)}=X_{(T, \lambda)}$.

Next, we summarize some useful properties of least-resolved trees that will be used repeatedly in the following sections.

Lemma 11. Let $(T, \lambda)$ be a phylogenetic tree that explains $X$. The following three conditions are equivalent:

1. $(T, \lambda)$ is least-resolved tree w.r.t. $X$.

2. Every edge of $(T, \lambda)$ is relevant and all inner edges are 1-edges.

3. (a) Every inner edge of $(T, \lambda)$ is a l-edge.

(b) For every inner edge $(u, v)$ there is an outer 0 -edge $(v, x)$ in $(T, \lambda)$.

Moreover, if $(T, \lambda)$ is least-resolved w.r.t. $X$, then

4. Any inner edge of $(T, \lambda)$ is distinguished by at least one informative rooted triple in $r_{I}(X)$, and

5. For any edge-contracted tree $\left(T_{e}, \lambda_{e}\right)$ of $(T, \lambda)$ there is a triple in $r_{I}(X)$ that is not displayed by $\left(T_{e}, \lambda_{e}\right)$, i.e., $(T, \lambda)$ is also least-resolved w.r.t. $r_{I}(X)$, and

6. The tree $\left(T(v), \lambda_{\mid C(v)}\right)$, that is, the subtree of $T$ rooted at the vertex $v$ with $\lambda_{\mid C(v)}(e)=\lambda(e)$ for any

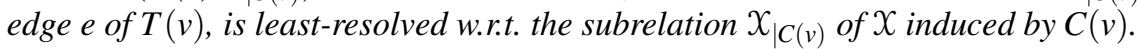

Proof. The equivalence of Conditions 1 and 2 is an immediate consequence of Lemma 7. Moreover, by Lemma 5, Condition 1 implies Condition 3(a). To see that also Condition 3(b) is implied given Conditions 1 or 2, observe that if $v$ is incident to 1 -edges only, then Lemma 6 implies that $(u, v)$ is irrelevant. Thus, $v$ must be incident to at least one 0 -edge. However, this 0 -edge cannot be an inner edge because inner 0 -edges can always be contracted due to Lemma 5. Thus, $v$ is incident to an outer 0-edge.

Now assume that Condition 3 is satisfied. First observe that none of the outer edges can be contracted without changing $X$. Let $(u, v)$ be an inner 1-edge and $(v, x)$ an outer 0 -edge. Since $(T, \lambda)$ is phylogenetic, there is a leaf $y$ for which $\operatorname{lca}(x, y)=u$. Thus, $(y, x) \in X$. However, contraction of the inner edge $(u, v)$ would yield $(y, x) \notin X$. Thus, none of the inner edges can be contracted and therefore, $(T, \lambda)$ is least-resolved w.r.t. $x$.

Property 4: Consider an arbitrary inner edge $e=(u, v)$ of $T$. Since $(T, \lambda)$ is phylogenetic, there are necessarily leaves $x, y$, and $z$ such that $\operatorname{lca}(x, y)=v$ and $\operatorname{lca}(x, y, z)=u$. Since $(u, v)$ is a 1-edge due to property 3 , the tree on $\{x, y, z\}$ displayed by $T$ must be one of $T_{3}, T_{5}, T_{7}, T_{10}, T_{11}$ or $T_{12}$ in Fig. 2 , where the red inner edge denotes the edge $(u, v)$. One easily checks explicitly that neither $T_{11}$ nor $T_{12}$ is least-resolved, since contraction of $e=(u, v)$ still yields $X_{\left(T_{e}, \lambda_{e}\right)}=X_{(T, \lambda)}$. The remaining trees $T_{3}, T_{5}, T_{7}$, and $T_{10}$, on the other hand, are informative triples $x y \mid z \in r_{I}(X)$. Since lca $(x, y)=v$ and lca $(x, y, z)=u$, the edge $e$ is by definition distinguished by the triple in $x y \mid z \in r_{I}(X)$. 
Property 5: Recall that each inner edge $e=(u, v)$ is distinguished by a triple $x y \mid z \in r_{I}(X)$; therefore $\operatorname{lca}(x, y)=v$ and lca $(x, y, z)=u$. However, contraction of $e$ would yield $\operatorname{lca}_{T_{e}}(x, y)=\operatorname{lca}_{T_{e}}(x, y, z)$, which in turn would imply that $x y \mid z \in r_{I}(X)$ is not displayed by $T_{e}$, a contradiction.

Property 6: By construction, no edge $(a, b)$ with $v \succeq_{T} a$ was removed in $T(v)$. Since $\lambda_{\mid C(v)}(e)=\lambda(e)$ for any edge $e$ of $T(v)$, Property 3 is trivially fulfilled in $\left(T(v), \lambda_{\mid C(v)}\right)$. Thus, $\left(T(v), \lambda_{\mid C(v)}\right)$ is least-resolved w.r.t. $X_{\mid C(v)}$.

As an immediate consequence of Lemma 9, which implies that all edge-contractions can be performed independently of each other, we can observe that for every edge-labeled tree $(T, \lambda)$ there exists a unique least-resolved tree $(\widehat{T}, \widehat{\lambda})$ that can be obtained from $(T, \lambda)$ by a sequence of edge-contractions. Every tree explaining $X$ is therefore a refinement of a least-resolved tree that explains $X$. By Lemma 4 , any tree that explains $\mathcal{X}$ must display the triples in $r_{I}(\mathcal{X})$. An even stronger result holds however:

Lemma 12. If $(T, \lambda)$ be a least-resolved tree w.r.t. $X=X_{(T, \lambda)}$, then $r_{I}(X)$ identifies $(T, \lambda)$.

Proof. If $r_{I}(X)=\emptyset$, then, by construction, all induced subgraphs on three vertices must be isomorphic to one of the graphs $A_{1}, A_{2}, A_{3}$, or $A_{4}$ in Fig. 2. In this case, $(T, \lambda)$ is a star-tree, i.e., an edge-labeled tree that consists of outer edges only. Otherwise, $(T, \lambda)$ contains inner edges that are, by Lemma 11, distinguished by at least one informative rooted triple in $r_{I}(X)$, contradicting that $r_{I}(X)=\emptyset$. Hence, $r(T)=\emptyset$, and therefore, $r(T)=\operatorname{cl}\left(r_{I}(X)\right)$. Lemma 1 implies that $r_{I}(X)$ identifies $(T, \lambda)$.

In the case $r_{I}(X) \neq \emptyset$, assume for contradiction that $r(T) \neq \operatorname{cl}\left(r_{I}(X)\right)$. By Lemma 4 we have $r_{I}(X) \subseteq$ $r(T)$. Isotony of the closure, Theorem 3.1(3) in [4], ensures $\operatorname{cl}\left(r_{I}(\mathcal{X})\right) \subseteq \operatorname{cl}(r(T))=r(T)$. Our assumption therefore implies $\operatorname{cl}\left(r_{I}(X)\right) \subsetneq r(T)$, and thus the existence of a triple $a b \mid c \in r(T) \backslash \operatorname{cl}\left(r_{I}(X)\right)$. In particular, therefore, $a b \mid c \notin r_{I}(\mathcal{X})$. Note that neither $a c \mid b$ nor $b c \mid a$ can be contained in $r_{I}(X)$, since $(T, \lambda)$ explains $X$ and, by assumption, already displays the triple $a b \mid c$. Thus, $r_{I}(X)$ contains no triples on $\{a, b, c\}$.

Lemma 11 implies that there exists a vertex $v \in \operatorname{child}(l c a(a, b, c))$, with $v \succeq l c a(a, b)$, and $(l c a(a, b, c), v)$ is a 1-edge. The subtree $T_{a b c}$ of $(T, \lambda)$ with leaves $a, b, c$ thus corresponds to one of $T_{3}, T_{5}, T_{7}, T_{10}, T_{11}$, or $T_{12}$ shown in Fig. 2. Recall that $T_{3}, T_{5}, T_{7}$, and $T_{10}$ explain the induced subgraphs $A_{5}, A_{6}, A_{7}$, and $A_{8}$, respectively. If $T_{a b c}$ is one of $T_{3}, T_{5}, T_{7}$, or $T_{10}$, then we would have a triple with leaves $a, b, c$ in $r_{I}(X)$. Since this is not the case by assumption, $T_{a b c}$ must be either $T_{11}$ or $T_{12}$. Thus, the subgraph of $X$ induced by $a, b, c$ is isomorphic to either $A_{1}$ or $A_{4}$.

Moreover, by Lemma 11, there must be a leaf $d \in \operatorname{child}(v)$ such that $(v, d)$ is a 0 -edge. Hence, the subtrees $T_{a c d}$ and $T_{b c d}$ with leaves $a, c, d$ and $b, c, d$, respectively, correspond to one the trees $T_{3}, T_{5}, T_{7}$, and $T_{10}$. Thus, the subgraph of $X$ induced by $a, c, d$ or $b, c, d$ must be isomorphic to a valid triangle $A_{5}, A_{6}, A_{7}$ or $A_{8}$. By construction, $a d \mid c \in r_{I}(X)$ and $b d \mid c \in r_{I}(X)$. Hence, any tree that explains $X$ must display $a d \mid c$ and $b d \mid c$. As shown in [9], a tree displaying $a d \mid c$ and $b d \mid c$ also displays $a b \mid c$. This implies, however, that $a b \mid c \in \operatorname{cl}\left(r_{I}(X)\right)$, a contradiction to our assumption.

Therefore, $\operatorname{cl}\left(R_{I}\right)=r(T)$ and we can finally apply Lemma 1 to conclude that $r_{I}(\mathcal{X})$ identifies $(T, \lambda)$.

We are now in the position to derive the main result of this section.

Theorem 1. Let $X \subseteq L \times L$ be a valid relation, $(T, \lambda)$ be a phylogenetic tree that explains $X$ and let $(\widehat{T}, \widehat{\lambda})$ be a least-resolved phylogenetic tree w.r.t. $X$. Then, $(T, \lambda)$ displays $(\widehat{T}, \widehat{\lambda})$. Moreover, the tree $(\widehat{T}, \widehat{\lambda})$ has the minimum number of vertices among all trees that explain $\mathcal{X}$, and is unique.

Proof. The first statement is an immediate consequence of Lemma 9. Lemma 12 implies that $r_{I}(X)$ identifies $(\widehat{T}, \widehat{\lambda})$. Hence, any tree that displays $r_{I}(X)$ is a refinement of $(\widehat{T}, \widehat{\lambda})$ and thus, must have more vertices. Lemma 12 also implies that $(T, \lambda)$ displays $(\widehat{T}, \widehat{\lambda})$. Moreover, Lemma 4 ensures that any tree explaining $X$ display $r_{I}(\mathcal{X})$. Combining these two observations, we conclude that $\widehat{T}$ has the minimum number of vertices among all trees that explain $X$.

By Lemma 11, all inner and outer edges of $(\widehat{T}, \widehat{\lambda})$ are relevant, and thus, their labels cannot be changed without changing $X$. Moreover, Lemma 10 implies that there is no further sequence of edge contractions that could be applied to $(\widehat{T}, \widehat{\lambda})$ to obtain another tree that explains $X$. Hence, $(\widehat{T}, \widehat{\lambda})$ is unique.

\section{Characterization of Valid Xenology Relations}

In this section we prove our main result: a binary relation $\mathcal{X}$ is explained by a tree if and only if it contains only valid triangles. The key idea of the proof, which proceeds by induction on the number of leaves, is to consider the superposition of trees explaining two induced subrelations, each of which is obtained by 
removing a single vertex from $\mathcal{X}$. We first establish several technical results for these trees. To this end we introduce some notation that will be used in this section only.

Definition 7. Let $(T, \lambda)$ be an edge-labeled phylogenetic tree and $e=(u, v)$ be an outer-edge of $T$. We write $\left(T-v, \lambda_{L-v}\right)$ for the tree obtained from $(T, \lambda)$ by removing the outer edge e and vertex $v$ from $T$ and keep the edge-labels of all remaining edges.

For an outer edge $e=(u, v)$ we therefore have $\left(T-v, \lambda_{\mid L-v}\right)=\left(T_{e}, \lambda_{e}\right)$ if and only if either $u=\rho_{T}$ and $\operatorname{deg}_{T-v}(u)>1$ or $u \neq \rho_{T}$ and $\operatorname{deg}_{T-v}(u)>2$.

Definition 8. Let $X \subset L \times L$ be an irreflexive relation and consider $l_{1}, \ldots, l_{k} \in L$. The set $X_{\neg l_{1}, \ldots, l_{k}}$ denotes the subrelation of $\mathcal{X}$ that is induced by $L \backslash\left\{l_{1}, \ldots, l_{k}\right\}$.

We emphasize that the results established in the previous sections are in general not valid for nonphylogenetic trees. Nevertheless, it is useful in the following to extend some concepts to more general trees. In particular, we say that an edge-labeled rooted (but possibly non-phylogenetic) tree $(T, \lambda)$ explains a given irreflexive relation $X$ if for any pair $(x, y) \in X$ there is a 1-edge on the path from lca $(x, y)$ to $y$.

Using the same arguments as in the proof of Lemma 2 we observe that $\left(T-v, \lambda_{\mid L-v}\right)$ explains $X_{\neg v}$.

Lemma 13. Let $(T, \lambda)$ be a least-resolved phylogenetic tree on L w.r.t. $X=X_{(T, \lambda)}$, and $v \in$ L. Let $\left(T^{\prime}, \lambda^{\prime}\right)$ be a least-resolved phylogenetic tree w.r.t. $X_{\neg v}$. Then, $\left(T^{\prime}, \lambda^{\prime}\right)$ is displayed by $\left(T-v, \lambda_{\mid L-v}\right)$. In particular, $\left(T^{\prime}, \lambda^{\prime}\right)=\left(T-v, \lambda_{\mid L-v}\right)$ if and only if $(i) \operatorname{par}(v)=\rho_{T}$ and $\operatorname{deg}_{T}\left(\rho_{T}\right)>2$ or $($ ii $) \operatorname{deg}_{T}(\operatorname{par}(v))>3$ and $\lambda_{\mid L-v}(\operatorname{par}(v), u)=0$ for some child $u \in \operatorname{child}(\operatorname{par}(v)), u \neq v$.

Proof. Let $\left(T^{\prime}, \lambda^{\prime}\right)$ be least-resolved w.r.t. $\chi_{\neg v}$. If $\left(T-v, \lambda_{L-v}\right)$ is phylogenetic, then we may apply Thm. 1 to verify that $\left(T^{\prime}, \lambda^{\prime}\right)$ is indeed displayed by $\left(T-v, \lambda_{\mid L-v}\right)$. Now assume that $\left(T-v, \lambda_{\mid L-v}\right)$ is not phylogenetic. In this case, either (a) $\operatorname{par}(v) \neq \rho_{T}$ is an inner vertex of degree 2 , or (b) the root $\rho$ of $T-v$ has degree 1 , and hence $\rho_{T}=\operatorname{par}(v)$.

Case (a): If $x=\operatorname{par}(v) \neq \rho_{T}$ is an inner vertex of degree 2 , let $T^{*}$ be the tree obtained by a simple contraction of the edge $(\operatorname{par}(x), x)$ and setting $\lambda_{\mid L-v}(x, \operatorname{child}(x))=1$. The labels of all other edges are kept. By construction, we obtain a phylogenetic tree $\left(T^{*}, \lambda^{*}\right)$ that still explains $X_{\neg v}$ and satisfies $\left(T^{\prime}, \lambda^{\prime}\right) \leq$ $\left(T^{*}, \lambda^{*}\right) \leq\left(T-v, \lambda_{\mid L-v}\right)$. Therefore, $\left(T^{\prime}, \lambda^{\prime}\right)$ is displayed by $\left(T-v, \lambda_{\mid L-v}\right)$.

Case (b): If the root $\rho$ of $T-v$ has degree 1 , let $T^{*}$ be the tree obtained by deleting $\rho$ and the edge $(\rho, w)$, where $w$ denotes the unique child of $\rho$ in $T-v$, and declaring child $(\rho)$ as the root of $T^{\prime}$. For all other edges set $\lambda^{*}(e)=\lambda_{L-v}(e)$. Again, we obtain a phylogenetic tree $\left(T^{*}, \lambda^{*}\right)$ that still explains $X_{\neg v}$. Repeating the arguments of Case $(a)$, we can conclude that $\left(T^{\prime}, \lambda^{\prime}\right)$ is displayed by $\left(T-v, \lambda_{\mid L-v}\right)$.

Now assume that $\left(T^{\prime}, \lambda^{\prime}\right)=\left(T-v, \lambda_{\mid L-v}\right)$. There are two cases: either $\operatorname{par}(v)$ is the root $\rho_{T}$ or not. If $\operatorname{par}(v)=\rho_{T}$, then $\operatorname{deg}_{T}\left(\rho_{T}\right) \leq 2$ would imply that $\operatorname{deg}_{T^{\prime}}\left(\rho_{T}\right) \leq 1$, in which case $\left(T^{\prime}, \lambda^{\prime}\right)$ would not be a phylogenetic tree; a contradiction, since $\left(T^{\prime}, \lambda^{\prime}\right)$ is phylogenetic. Hence, if $\operatorname{par}(v)=\rho_{T}$, then $\operatorname{deg}_{T}\left(\rho_{T}\right)>2$. Now assume that $\operatorname{par}(v) \neq \rho_{T}$. Thus, there is an inner edge $(x, \operatorname{par}(v))$ where $x=\operatorname{par}(\operatorname{par}(v))$. Lemma 11(3) implies that this edge $(x, \operatorname{par}(v))$ must be incident to an outer 0-edge in $\left(T^{\prime}, \lambda^{\prime}\right)$ and hence, $\lambda_{\mid L-v}(\operatorname{par}(v), u)=0$ for some leaf $u \in L \backslash\{v\}$. Moreover, as $\left(T^{\prime}, \lambda^{\prime}\right)$ is phylogenetic, $\operatorname{deg}_{T-v}(\operatorname{par}(v))>2$ and hence, $\operatorname{deg}_{T}(\operatorname{par}(v))>3$.

Conversely, assume first that $\operatorname{par}(v)=\rho_{T}$ and $\operatorname{deg}_{T}\left(\rho_{T}\right)>2$. In this case, $\left(T-v, \lambda_{L-v}\right)$ is still a phylogenetic tree. By construction, $E^{0}(T-v)=E^{0}(T)$ and $\lambda_{L-v}(e)=\lambda(e)$ for all $e \in E^{0}(T-v)$ Thus, any inner edge of $T-v$ is a 1-edge. Lemma 11(3) implies that for each inner edge $e=(x, y)$ in $T$ there is an outer 0 -edge $(y, z)$ in $(T, \lambda)$. This property still holds in $\left(T-v, \lambda_{\mid L-v}\right)$ because the deleted edge $(\operatorname{par}(v), v)$ is incident to the root of $(T, \lambda)$. Thus all edges of $\left(T-v, \lambda_{\mid L-v}\right)$ are relevant. Lemma 11 implies that $\left(T-v, \lambda_{\mid L-v}\right)$ is least-resolved.

Now assume that $\operatorname{par}(v) \neq \rho_{T}$ and $\operatorname{deg}_{T}(\operatorname{par}(v))>3$. Thus, $\left(T-v, \lambda_{L-v}\right)$ is still a phylogenetic tree. Let $\lambda_{\mid L-v}(\operatorname{par}(v), u)=0$ for some child $u \in \operatorname{child}(\operatorname{par}(v)), u \neq v$. Now, we can apply similar arguments as above to conclude that all edges in $\left(T-v, \lambda_{L-v}\right)$ are relevant, and thus, $\left(T-v, \lambda_{\mid L-v}\right)$ is least-resolved.

In summary, if $\operatorname{par}(v)=\rho_{T}$ and $\operatorname{deg}_{T}\left(\rho_{T}\right)>2$ or $\lambda_{\mid L-v}(\operatorname{par}(v), u)=0$ for some child $u \in \operatorname{child}(\operatorname{par}(v))$, $u \neq v$, and $\operatorname{deg}_{T}(\operatorname{par}(v))>3$, then $\left(T-v, \lambda_{\mid L-v}\right)$ is least-resolved w.r.t. $X_{\neg v}$. By Thm. $1,\left(T-v, \lambda_{\mid L-v}\right)=$ $\left(T^{\prime}, \lambda^{\prime}\right)$.

An immediate consequence of Lemma 13 is the following result that is crucial for proving the main result.

Lemma 14. Let $(T, \lambda)$ and $\left(T-v, \lambda_{\mid L-v}\right)$ be defined as in Lemma 13, and $\left(T^{\prime}, \lambda^{\prime}\right)$ be the least-resolved phylogenetic tree that explains $X_{\neg v}$. Then, either

1. $\left(T-v, \lambda_{L-v}\right)=\left(T^{\prime}, \lambda^{\prime}\right)$, or

2. $\left(T^{\prime}, \lambda^{\prime}\right)$ is obtained from $\left(T-v, \lambda_{\mid L-v}\right)$ by a simple contraction of either 
(i) the inner edge $\left(\rho_{T}, u\right) \in E(T-v)$, in case that $\operatorname{par}(v)=\rho_{T}$ and $\operatorname{deg}_{T}\left(\rho_{T}\right)=2$, or

(ii) the inner edge $(\operatorname{par}(x), x) \in E(T-v)$, where $x=\operatorname{par}(v) \neq \rho_{T}$, and setting $\lambda^{\prime}(x, \operatorname{child}(x))=1$, otherwise.

In either case $\lambda^{\prime}(e)=\lambda_{\mid L-v}(e)$ for all non-contracted edges $e$.

In particular, $\left(T-v, \lambda_{\mid L-v}\right)$ displays the least-resolved phylogenetic tree $\left(T^{\prime}, \lambda^{\prime}\right)$ that explains $X_{\neg v}$ and therefore, $r\left(T^{\prime}\right) \subseteq r(T-v)$.

Proof. By Lemma 13, $\left(T-v, \lambda_{\mid L-v}\right)$ is least-resolved if and only if $\operatorname{par}(v)=\rho_{T}$ and $\operatorname{deg}_{T}\left(\rho_{T}\right)>2$ or there exists a leaf $u \in \operatorname{par}(v), u \neq v$, such that $\lambda_{\mid L-v}(\operatorname{par}(v), u)=0$ and $\operatorname{deg}_{T}(\operatorname{par}(v))>3$. If $\left(T-v, \lambda_{\mid L-v}\right)$ is not least-resolved and $\operatorname{par}(v)=\rho_{T}$, we have $\operatorname{deg}_{T-v}\left(\rho_{T}\right)=1$. Due to Lemma 11(6), the tree $\left(T^{\prime}, \lambda^{\prime}\right)$ obtained by a simple contraction of the single edge $\left(\rho_{T}, u\right)$ and adopting $u$ as the new root is least-resolved w.r.t. $X_{\neg v}$.

If $\left(T-v, \lambda_{L-v}\right)$ is not least-resolved and $\operatorname{par}(v) \neq \rho_{T}$, then either (a) there is no leaf $u \in \operatorname{child}(\operatorname{par}(v))$, $u \neq v$, with $\lambda_{L-v}(\operatorname{par}(v), u)=0$ or $(\mathrm{b}) \operatorname{deg}_{T-v}(\operatorname{par}(v))=2$. Indeed, $\operatorname{deg}_{T-v}(\operatorname{par}(v))>2$ and $u \in$ $\operatorname{child}(\operatorname{par}(v))$ with $\lambda_{\mid L-v}(\operatorname{par}(v), u)=0$ implies that $\left(T-v, \lambda_{L-v}\right)$ is least-resolved. On the other hand, $\operatorname{deg}_{T-v}(\operatorname{par}(v)) \geq 2$ because $T$ is phylogenetic.

Case (a). Assume that $(\operatorname{par}(v), u)$ is a 1-edge for all children $u \neq v$ of $\operatorname{par}(v)$. Then, the inner edge $(\operatorname{par}(x), x) \in E(T-v)$ is irrelevant in $\left(T-v, \lambda_{\mid L-v}\right)$; thus it can be contracted. Since $(T, \lambda)$ is least-resolved, Lemma 11(3) ensures that every inner vertex in $\left(T-v, \lambda_{\mid L-v}\right)$ other than $\operatorname{par}(v)$ is adjacent to an outer 0 -edge. Hence, contraction of $(x, \operatorname{par}(v))$ in $(T-v, \lambda-v)$ yields the least-resolved tree w.r.t. $X_{\neg v}$.

Case (b). If $\operatorname{deg}_{T-v}(\operatorname{par}(v))=2$ and $\lambda_{\mid L-v}(\operatorname{par}(v), u)=1$, the edge $(\operatorname{par}(x), x)$ can be contracted without changing the relation and similar arguments as in case (a) show that $\left(T-v, \lambda_{\mid L-v}\right)$ is least-resolved w.r.t. $X_{\neg v}$. If $\lambda_{\mid L-v}(\operatorname{par}(v), u)=0$, then the construction as in 2.(ii) does not change $X$ since $\lambda(\operatorname{par}(x), x)=1$. Again, similar arguments as in case (a) ensure that $\left(T-v, \lambda_{L-v}\right)$ is least-resolved w.r.t. $X_{\neg v}$.

Obviously, either $\left(T^{\prime}, \lambda^{\prime}\right)=\left(T-v, \lambda_{\mid L-v}\right)$ or $\left(T^{\prime}, \lambda^{\prime}\right)$ can be obtained from $\left(T-v, \lambda_{\mid L-v}\right)$ by a single simple edge-contraction. Thus $\left(T^{\prime}, \lambda^{\prime}\right)$ is displayed by $\left(T-v, \lambda_{\mid L-v}\right)$ and $r\left(T^{\prime}\right) \subseteq r(T-v)$.

Let $(T=(V, E), \lambda)$ be an edge-labeled phylogenetic tree. Moreover, let $(x, y) \in E$ and let $\left(T_{e}, \lambda_{e}\right)$ be the phylogenetic tree obtained from $(T, \lambda)$ by extended contraction of $e$ in $(T, \lambda)$. Given $\left(T_{e}, \lambda_{e}\right)$ it is possible to recover the tree $(T, \lambda)$ reverting the extended contraction of $e$. If $e$ was an internal edge, this amounts to subdividing a vertex $z$, yielding $e=(u, v)$, and a bi-partitioning of the set of children of $z$ into the children of $u$ and $v$. If $e$ was an external edge incident to a degree 2 node, an edge $f$ in $\left(T_{e}, \lambda_{e}\right)$ is subdivided and $e$ is attached to the new inner vertex. In addition, the labeling is adjusted. We refer to these constructions as reinsertion of e into $\left(T_{e}, \lambda_{e}\right)$.

Lemma 15. Given a Fitch relation $X$ such that $X_{\neg u}, X_{\neg v}$, and $X_{\neg u v}$ are valid for some $u, v \in V(X)$. Let $\left(T_{\neg u}, \lambda_{\neg u}\right),\left(T_{\neg v}, \lambda_{\neg v}\right)$ and $\left(T_{\neg u v}, \lambda_{\neg u v}\right)$ be the least-resolved trees that explain $\mathcal{X}_{\neg u}, X_{\neg v}$, and $X_{\neg u v}$, respectively.

Then, there is a tree $(T, \lambda)$ that correctly explains all members in $X \backslash X[u, v]$, i.e., $X_{(T, \lambda)}[x, y]=X[x, y]$ for all $x, y$ with $\{x, y\} \neq\{u, v\}$. Moreover $(T, \lambda)$ displays $\left(T_{\neg u}, \lambda_{\neg u}\right),\left(T_{\neg v}, \lambda_{\neg v}\right)$ and $\left(T_{\neg u v}, \lambda_{\neg u v}\right)$.

Proof. Consider the least-resolved tree $\left(T_{\neg u v}, \lambda_{\neg u v}\right)$ that correctly explains $X_{\neg u v}$. By Lemma 14, this tree can be obtained from the least-resolved trees $\left(T_{\neg u}, \lambda_{\neg u}\right)$ and $\left(T_{\neg v}, \lambda_{\neg v}\right)$ by removing the vertices $v$ and $u$, respectively, and possibly contraction of edges. More precisely, $\left(T_{\neg u v}, \lambda_{\neg u v}\right)=\left(T_{\neg u}-v, \lambda_{\neg u \mid L^{\prime}}\right)$ or $\left(T_{\neg u v}, \lambda_{\neg u v}\right)$ is obtained from $\left(T_{\neg u}-v, \lambda_{\neg u \mid L^{\prime}}\right)$ by contracting exactly the edge $(x, y)$ where $y=\operatorname{par}(v)$ and a possible relabeling of the children of $y$. In what follows, we denote by $x y$ the vertex in $T_{u v}$ that is obtained by contraction of this edge $(x, y)$. In the same way, $\left(T_{\neg u v}, \lambda_{\neg u v}\right)$ is obtained from $\left(T_{\neg v}-u, \lambda_{\neg v \mid L^{\prime}}\right)$ and if the edge $\left(x^{\prime}, y^{\prime}\right)$ was contracted, then $x^{\prime} y^{\prime}$ denotes the resulting vertex in $T_{\neg u v}$.

Therefore, the following cases must be considered:

1. $\left(T_{\neg u v}, \lambda_{\neg u v}\right)=\left(T_{\neg u}-v, \lambda_{\neg u \mid L^{\prime}}\right)=\left(T_{\neg v}-u, \lambda_{\neg v \mid L^{\prime}}\right)$.

2. Either

(a) $\left(T_{\neg u v}, \lambda_{\neg u v}\right)=\left(T_{\neg u}-v, \lambda_{\neg u \mid L^{\prime}}\right) \lesseqgtr\left(T_{\neg v}-u, \lambda_{\neg v \mid L^{\prime}}\right)$, or

(b) $\left(T_{\neg u v}, \lambda_{\neg u v}\right)=\left(T_{\neg v}-u, \lambda_{\neg v \mid L^{\prime}}\right) \lesseqgtr\left(T_{\neg u}-v, \lambda_{\neg u \mid L^{\prime}}\right)$.

3. $\left(T_{\neg u v}, \lambda_{\neg u v}\right) \lesseqgtr\left(T_{\neg u}-v, \lambda_{\neg u \mid L^{\prime}}\right)$ and $\left(T_{\neg u v}, \lambda_{\neg u v}\right) \lesseqgtr\left(T_{\neg v}-u, \lambda_{\neg v \mid L^{\prime}}\right)$ and either

(a) $x y \neq x^{\prime} y^{\prime}$ or (b) $x y=x^{\prime} y^{\prime}$.

In Case 1, one can simply add the edge $(\operatorname{par}(v), v)$ and $(\operatorname{par}(u), u)$ together with the original edge labels $\lambda_{\neg u}(\operatorname{par}(v), v)$ and $\lambda_{\neg v}(\operatorname{par}(u), u)$ to obtain a tree $(T, \lambda)$ that contains both $\left(T_{\neg u}, \lambda_{\neg u}\right)$ and $\left(T_{\neg v}, \lambda_{\neg v}\right)$ as subtrees and thus, $\mathcal{X}_{(T, \lambda)}[x, y]=X[x, y]$ for all $x, y$ with $\{x, y\} \neq\{u, v\}$. 
In Case 2(a), one can simply add the edge $(\operatorname{par}(v), v)$ together with the original edge label $\lambda_{\neg u}(\operatorname{par}(v), v)$ to obtain $\left(T_{\neg u}, \lambda_{\neg u}\right)$. Since $x^{\prime} y^{\prime}$ denotes the vertex that results from contracting the edge $\left(x^{\prime}, y^{\prime}\right)$ in $\left(T_{\neg v}-\right.$ $\left.u, \lambda_{\neg v \mid L^{\prime}}\right)$, this vertex is also contained in $\left(T_{\neg u}, \lambda_{\neg u}\right)$. Now, we reinsert $x^{\prime} y^{\prime}$ such that we obtain a tree $(T, \lambda)$ that contains $\left(T_{\neg v}, \lambda_{\neg v}\right)$ as a subtree. Hence, $X_{\neg u v}$ and $X_{\neg v}$ are correctly explained by $(T, \lambda)$. It remains to show that also all $\mathcal{X}[v, z]$ and $\mathcal{X}[z, v]$ with $z \neq u$ are still correctly explained. Assume for contradiction that this is not the case and that $\mathcal{X}[v, z] \neq \mathcal{X}_{(T, \lambda)}[v, z]$ for some $z \neq u$. This is only possible if in the tree $(T, \lambda)$ there is this 1-edge $\left(x^{\prime}, y^{\prime}\right)$ contained in the path from $\operatorname{lca}_{T}(v, z)$ to $z$. Hence, $X_{(T, \lambda)}[v, z]=(v, z)$, which implies that the path from $\operatorname{lca}_{T_{\neg u}}(v, z)$ to $z$ contains only 0-edges. Moreover, $T_{\neg u}$ is least-resolved w.r.t. $X_{\neg u}$. Hence, all inner edges are 1-edges. Therefore, $\operatorname{lca}_{T_{\neg u}}(v, z)=x^{\prime} y^{\prime}$ and $\left(x^{\prime} y^{\prime}, z\right) \in E\left(T_{\neg u}\right)$ must be an outer 0-edge. Note that this implies that $z$ is a child of $y^{\prime}$ in $T_{\neg v}$. By construction according to Lemma 14(ii), we have contracted the edge $\left(x^{\prime}, y^{\prime}\right)$ in $\left(T_{\neg v}-u, \lambda_{\mid L-u}\right)$ and relabeled all outer edges in $T_{\neg v}$ incident to $y^{\prime}$ as 1-edges. But this implies that $\left(x^{\prime} y^{\prime}, z\right)$ is a 1-edge in $T_{\neg u}$; a contradiction. The assumption $X[z, v] \neq \mathcal{X}_{(T, \lambda)}[z, v]$ for some $z \neq u$ yields a contradiction using analogous arguments.

Case 2(b) is settled by interchanging the roles of $u$ and $v$ in Case 2(a).
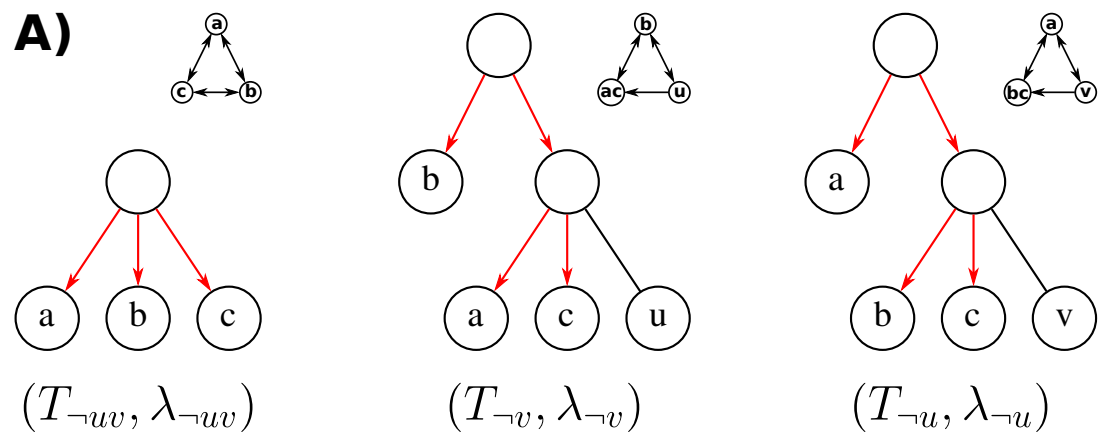

B)

$\left(T_{\neg u v}, \lambda_{\neg u v}\right)$
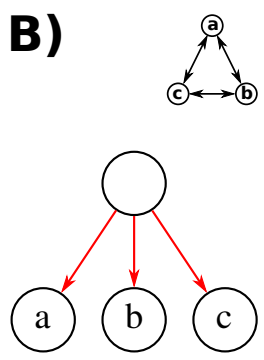

$\left(T_{\neg u v}, \lambda_{\neg u v}\right)$
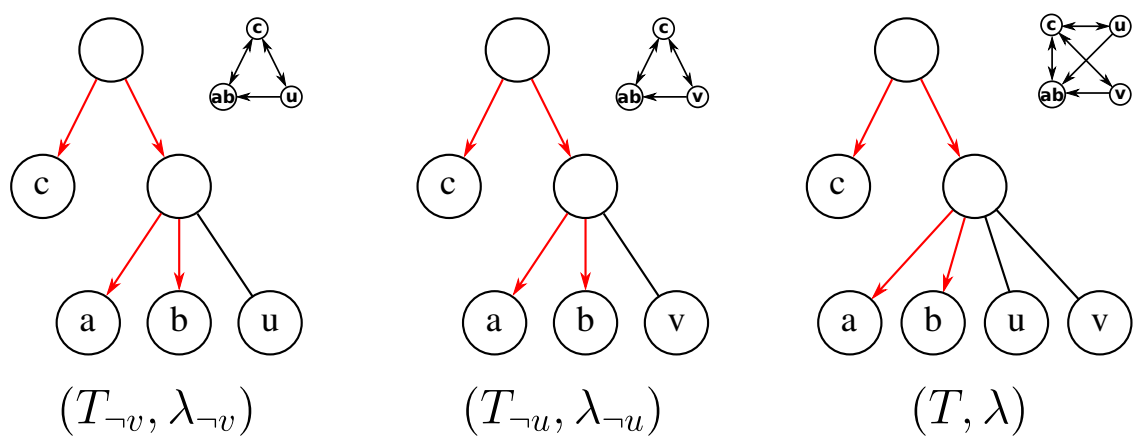

Figure 4: (A) The two least-resolved trees $\left(T_{\neg u}, \lambda_{\neg u}\right)$ and $\left(T_{\neg v}, \lambda_{\neg v}\right)$ that explain $X_{\neg u}$ and $X_{\neg v}$ respectively both explain the least-resolved tree $\left(T_{\neg u v}, \lambda_{\neg u v}\right)$ that explains $\mathcal{X}_{\neg u v}$. However, there exists no tree $(T, \lambda)$ that explains $X$, thus there is no valid Fitch relation $X$ that contains both $X_{\neg u}$ and $X_{\neg \nu}$. This is due to the fact that the triples $a c \mid b$ and $b c \mid a$ in $\left(T_{\neg u}, \lambda_{\neg u}\right)$ and $\left(T_{\neg v}, \lambda_{\neg v}\right)$ contradict each other. (B) We have $\mathcal{C}_{x}=\{c\}=\mathcal{C}_{x^{\prime}}$ and $\mathcal{C}_{y}=\{a, b\}=\mathcal{C}_{y^{\prime}}$ in the least-resolved trees $\left(T_{\neg u}, \lambda_{\neg u}\right)$ and $\left(T_{\neg v}, \lambda_{\neg v}\right)$. In this case, there exists a tree $(T, \lambda)$ that displays $\left(T_{\neg u v}, \lambda_{\neg u v}\right)$, $\left(T_{\neg u}, \lambda_{\neg u}\right)$ and $\left(T_{\neg v}, \lambda_{\neg v}\right)$, and explains $X$. The Fitch relation corresponding to each tree is shown in the upper right corner. Two nodes $x$ and $y$ are represented as one node $x y$ if they have the same relationship with every other node.

Case 3. In order to obtain $(T, \lambda)$ from $\left(T_{\neg u v}, \lambda_{\neg u v}\right)$, we need to undo the contractions that lead to $x y$ and $x^{\prime} y^{\prime}$ and in addition, reinsert the edges $\left(y^{\prime}, u\right)$ and $(y, v)$ with original edge-labeling such that $(T, \lambda)$ contains both $\left(T_{\neg u}, \lambda_{\neg u}\right)$ and $\left(T_{\neg v}, \lambda_{\neg v}\right)$ as subtrees and thus, $\mathcal{X}_{(T, \lambda)}[x, y]=X[x, y]$ for all $x, y$ with $\{x, y\} \neq\{u, v\}$. The subdivision of $x y$ partitions the set of children child $(x y)$ of the vertex $x y$ into two disjoint sets $\mathcal{C}_{x}$ and $\mathcal{C}_{y}$ in such a way that $\mathcal{C}_{x}$ contains all children of $x$ that are distinct from $y$ and $\mathcal{C}_{y}$ contains all children of $y$ in $\left(T_{\neg u}-v, \lambda_{\neg u \mid L^{\prime}}\right)$. Analogously, the sets $\mathcal{C}_{x^{\prime}}$ and $\mathcal{C}_{y^{\prime}}$ are obtained by partitioning $\operatorname{child}\left(x^{\prime} y^{\prime}\right)$ in $\left(T_{\neg v}-u, \lambda_{\neg v \mid L^{\prime}}\right)$. The sets $\mathcal{C}_{x}, \mathcal{C}_{x^{\prime}}, \mathcal{C}_{y}$, and $\mathcal{C}_{y^{\prime}}$ are all non-empty because $\left(T_{\neg u}, \lambda_{\neg u}\right)$ and $\left(T_{\neg v}, \lambda_{\neg v}\right)$ are phylogenetic.

Case 3(a). $x y \neq x^{\prime} y^{\prime}$. By definition of $\left(T_{\neg u v}, \lambda_{\neg u v}\right)$, it is possible to subdivide $x y$ and add $(\operatorname{par}(v), v)$ with the edge-labeling $\lambda_{\neg u}(\operatorname{par}(v), v)$ such that we obtain $\left(T_{\neg u}, \lambda_{\neg u}\right)$. Subdivision of $x^{\prime} y^{\prime}$ in $\left(T_{\neg u}, \lambda_{\neg u}\right)$ results in a tree $(T, \lambda)$ that contains $\left(T_{\neg v}, \lambda_{\neg v}\right)$ as a subtree. Hence, $(T, \lambda)$ correctly explains $X_{\neg u v}$ and $X_{\neg v}$. Arguments analogous to Case 2 now show that $X[z, v]$ and $X[v, z]$ are correctly explained for any $z \neq u$, thus $(T, \lambda)$ correctly explains $X_{\neg u}$.

Case 3(b). $x y=x^{\prime} y^{\prime}$. Since $x y=x^{\prime} y^{\prime},(T, \lambda)$ is obtained from $\left(T_{\neg u v}, \lambda_{\neg u v}\right)$ by reinsertion of a single edge. 
To ensure that $(T, \lambda)$ displays both $\left(T_{\neg u}, \lambda_{\neg u}\right)$ and $\left(T_{\neg v}, \lambda_{\neg v}\right)$, we need to show that $\mathcal{C}_{x}=\mathcal{C}_{x^{\prime}}$ and $\mathcal{C}_{y}=\mathcal{C}_{y^{\prime}}$.

First, we show that all 0 -edges incident to $x y$ in $\left(T_{\neg u v}, \lambda_{\neg u v}\right)$ are incident to $x$ and $x^{\prime}$ in $\left(T_{\neg u}, \lambda_{\neg u}\right)$ and $\left(T_{\neg v}, \lambda_{\neg v}\right)$, respectively. Let $M$ denote the set of all leaves $z \in \operatorname{child}(x y)$ for which $\lambda^{\prime}(x y, z)=0$ in $T_{\neg u v}$. Since $\left(T_{\neg u v}, \lambda_{\neg u v}\right)$ is least-resolved, $M \neq \emptyset$. For any $w \in \operatorname{child}(x y)$, and $z \in M$ there is no 1-edge on the path from lca $(w, z)$ to $z$ in $\left(T_{\neg u v}, \lambda_{\neg u v}\right)$. We proceed by showing that $M \subseteq \mathcal{C}_{x} \cap \mathcal{C}_{x^{\prime}}$. Assume for contradiction that $z \in \mathcal{C}_{x}$ but $z \notin \mathcal{C}_{x^{\prime}}$. Thus $z \in \mathcal{C}_{y^{\prime}}$. Furthermore, for any $w^{\prime} \in \mathcal{C}_{x^{\prime}}$, the 1-edge $e^{\prime}=\left(x^{\prime}, y^{\prime}\right)$ is contained in the path from lca $\left(w^{\prime}, z\right)$ to $z$ in the tree $\left(T_{\neg v}-u, \lambda_{v \mid L^{\prime}}\right)$. Since $\left(T_{\neg v}-u, \lambda_{v \mid L^{\prime}}\right)$ is phylogenetic, $\mathcal{C}_{x^{\prime}}$ is non-empty, i.e., such a $w^{\prime}$ exists. In contrast, for any $w \in \mathcal{C}_{x} \cup \mathcal{C}_{y}, w \neq z$, there is no 1-edge on the path from lca $(w, z)$ to $z$ in $\left(T_{\neg u}-v, \lambda_{u \mid L^{\prime}}\right)$. Since $\mathcal{C}_{x^{\prime}} \subseteq \mathcal{C}_{x} \cup \mathcal{C}_{y}$, the two trees $\left(T_{\neg u}-v, \lambda_{u \mid L^{\prime}}\right)$ and $\left(T_{\neg v}-u, \lambda_{v \mid L^{\prime}}\right)$ cannot explain the same relation $X_{\neg u v}$; this is the desired contradiction.

Hence, it remains to show that for every 1-edge $(x y, a)$ in $\left(T_{\neg u v}, \lambda \neg \neg v\right)$ either $a \in \mathcal{C}_{x} \cap \mathcal{C}_{x^{\prime}}$ or $a \in \mathcal{C}_{y} \cap \mathcal{C}_{y^{\prime}}$ is true. Assume for contradiction that $a \in \mathcal{C}_{x}$ but $a \notin \mathcal{C}_{x^{\prime}}$, i.e., $a \notin \mathcal{C}_{y}$ and $a \in \mathcal{C}_{y^{\prime}}$. This implies $[a, v] \in \mathcal{X}_{\neg u}$ and $(u, a) \in X_{\neg v}$. Since $\{u, v, a\}$ must form a valid triangle, either $(u, v) \in X$ or $[u, v] \in X$ must be true. On the other hand, since $M \subseteq \mathcal{C}_{x} \cap \mathcal{C}_{x^{\prime}}$ and the trees $\left(T_{\neg u}, \lambda_{\neg u}\right)$ and $\left(T_{\neg v}, \lambda_{\neg v}\right)$ are least-resolved, both $(y, v)$ and $\left(y^{\prime}, u\right)$ must be 0 -edges. By construction, $(T, \lambda)$ is obtained by reinserting a single edge in $\left(T_{\neg u v}, \lambda_{\neg u v}\right)$ in such a way that $\operatorname{par}(u)=\operatorname{par}(v)$. Thus we must have $u \mid v$; a contradiction, and we can conclude $\mathcal{C}_{y}=\mathcal{C}_{y^{\prime}}$ and $\mathcal{C}_{x}=\mathcal{C}_{x^{\prime}}$

We remark that the existence of the tree $(T, \lambda)$ asserted in Lemma 15 does not follow from the fact that both $\left(T_{\neg u}, \lambda_{\neg u}\right)$ and $\left(T_{\neg v}, \lambda_{\neg v}\right)$ explain $\left(T_{\neg u v}, \lambda_{\neg u v}\right)$. A counter-example is given in Fig. 4 . The condition that the trees together explain a Fitch relation cannot be relaxed in the proof.

Theorem 2. An irreflexive relation $X$ on $L$ is valid if and only if it is a Fitch relation.

Proof. Assume that $X$ is valid. Hence, there is a tree $(T, \lambda)$ that explains $X$. Let $x, y, z \in L$ be distinct vertices. Clearly, any subtree $T^{\prime} \subseteq T$ with leaf set $\{x, y, z\}$ must correspond to one of the trees $T_{1}, \ldots, T_{16}$ in Fig. 2. Since these subtrees can only encode the valid triangles $A_{1}, \ldots A_{8}$, the subgraph induced by $x, y, z$ in $X$ must be isomorphic to one of $A_{1}, \ldots A_{8}$. Since this statement is true for any three distinct vertices in $\mathcal{X}$, all triangles in $X$ are valid. Hence, $X$ is a Fitch relation.

Now assume that $X$ is a Fitch relation. The trivial relation on $L$, corresponding to the empty graph, is explained by any tree with leaf set $L$ that has only 0 -edges. For the non-trivial case we proceed by induction w.r.t. the number of vertices $|L|$. The base case consists of the valid triangles, for which the statement is trivially true. Assume now that all Fitch relations with $|L| \leq n$ are valid.

Let $X$ be a Fitch relation on $|L|=n+1$ vertices and let $u, v \in L$ be two distinct, arbitrarily chosen vertices. Clearly, $X_{\neg u}, X_{\neg v}$, and $X_{\neg u v}$ are Fitch relations and, by assumption, also valid. In particular, there are unique least-resolved trees $\left(T_{\neg u}, \lambda_{\neg u}\right),\left(T_{\neg v}, \lambda_{\neg v}\right)$ and $\left(T_{\neg u v}, \lambda_{\neg u v}\right)$ that explain $X_{\neg u}, X_{\neg v}$ and $X_{\neg u v}$, respectively. With the exception of the relation between $u$ and $v, X$ is therefore determined by $\left(T_{\neg u}, \lambda_{\neg u}\right)$ and $\left(T_{\neg v}, \lambda_{\neg v}\right)$, i.e., any pair $(x, y) \in X$ for which $\{x, y\} \neq\{u, v\}$ is explained by $\left(T_{\neg u}, \lambda_{\neg u}\right)$ or $\left(T_{\neg v}, \lambda_{\neg v}\right)$. In particular all pairs $(x, u)$ or $(u, x)$ in $X \backslash X[u, v]$ are explained by $\left(T_{\neg v}, \lambda_{\neg v}\right)$ and all pairs $(x, v)$ or $(v, x)$ in $X \backslash X[u, v]$ are explained by $\left(T_{\neg u}, \lambda_{\neg u}\right)$.

Lemma 15 implies that there is a tree that correctly explains all pairs in $X \backslash X[u, v]$ and displays $\left(T_{\neg u}, \lambda_{\neg u}\right),\left(T_{\neg v}, \lambda_{\neg v}\right)$ and $\left(T_{\neg u v}, \lambda_{\neg u v}\right)$. Thus, there is in particular a least-resolved tree $(T, \lambda)$ that fulfills these requirements.

$\mathcal{X}[u, v]$ is in some cases uniquely determined by $\mathcal{X} \backslash X[u, v]$ and the requirement that $\{u, v, x\}$ forms a valid triangle. The existence of $(T, \lambda)$ then implies immediately that $\mathcal{X}[u, v]$, and hence $\mathcal{X}$, is explained by $(T, \lambda)$.

This is not always the case, however. If more than one choice of $\mathcal{X}[u, v]$ completes $\mathcal{X} \backslash X[u, v]$, we need to show that a $(T, \lambda)$ exists for each of the possible choices. Denote by $\Delta_{u v}$ the set of triangles in $\mathcal{X}$ that contain $u$ and $v$. Full enumeration (which we leave to the reader) shows that $\mathcal{X}[u, v]$ is not uniquely determined if and only if all triangles in $\Delta_{u v}$ are of the form $A, B, C$ or $D$ listed in Fig. 5. Only certain combinations of these triangle types can occur: The co-occurence of $A$ and $B$ implies $(u, v) \in X$, hence $X[u, v]$ is uniquely determined, and hence $(T, \lambda)$ is also unique. The remaining cases can be classified as follows:

1. $\Delta_{u v}$ contains at least one triangle either of types $A, C$ and $D$ but not $B$, or of types $B, C$ and $D$ but not A.

2. $\Delta_{u v}$ consists of triangles of exactly one of the types $A, B$ and $C, D$, respectively, and for each type there is a triangle.

3. $\Delta_{u v}$ consists exclusively of triangles of the types $C$ and $D$ and for each type $C, D$ there is a triangle.

4. All triangles in $\Delta_{u v}$ are of the same type. 
A)

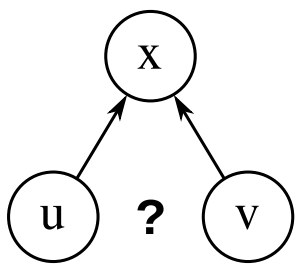

$u \mid v$

$(u, v) \in \mathcal{X}$

$(v, u) \in \mathcal{X}$
B)

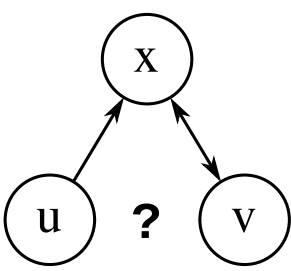

$(u, v) \in \mathcal{X}$

$[u, v] \in \mathcal{X}$
C)

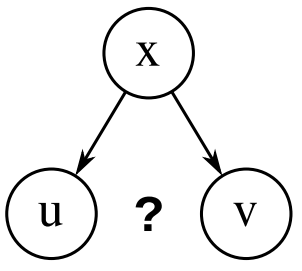

$u \mid v$

$(u, v) \in \mathcal{X}$

$(v, u) \in \mathcal{X}$

$[u, v] \in \mathcal{X}$
D)

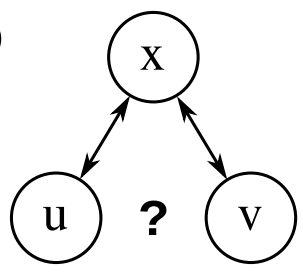

$u \mid v$

$(u, v) \in \mathcal{X}$

$(v, u) \in \mathcal{X}$

$[u, v] \in \mathcal{X}$

Figure 5: All cases where the relationship $X[u, v]$ cannot be uniquely inferred from $X \backslash X[u, v]$.

In each of these cases, there is more than one possible choice for $\mathcal{X}[u, v]$. Lemma 15 ensures that there is a least-resolved tree $(T, \lambda)$ that explains at least one of these choices. Given $(T, \lambda)$ for one particular choice, we show below that it is always possible to transform $(T, \lambda)$ into another least-resolved tree that explains $X$ with a different choice of $\mathcal{X}[u, v]$. The resulting tree $\left(T^{\prime}, \lambda^{\prime}\right)$ is unique by Thm. 1 , and thus the transformation can be inverted in a uniquely defined manner.

In what follows, we call $v \in L(T)$ a sink (resp. source) if for all $x \in L(T)$ we have $(x, v) \in X_{(T, \lambda)}$ (resp. $\left.(v, x) \in X_{(T, \lambda)}\right)$. Moreover, in order to exclude the trivial case $\Delta_{u v}=\emptyset$, we assume that $|L(T)| \geq 3$.

Case 1a. Suppose that $\Delta_{u v}$ contains at least one triangle each of types $A, C$ and $D$ but not of type $B$. Hence, $\mathcal{X}[u, v] \in\{(u, v),(v, u), u \mid v\}$. Thus, we show that for each of these choices of $X[u, v]$ there is a least-resolved tree that explains $X$.

Suppose that $(T, \lambda)$ explains $u \mid v$. Since all inner edges of $(T, \lambda)$ are 1-edges, $u$ and $v$ must be siblings and in particular, the edges $(\operatorname{lca}(u, v), v)$ and $(\operatorname{lca}(u, v), u)$ are 0 -edges. Since there is a triangle of each of the types $A, C$ and $D$, there is no leaf $x \in L(T) \backslash\{u, v\}$ with $x \mid u$ or $x \mid v$. Moreover, if there would be another vertex $x \in V(T) \backslash\{u, v\}$ that is adjacent to $\operatorname{lca}_{T}(u, v)$, then $(\operatorname{lca}(u, v), x)$ must be a 1-edge. Let $T^{*}$ denote the subtree of $T$ with root lca $(u, v)$ without the leaves $u$ and $v$. Therefore, $(T, \lambda)$ locally looks like the tree shown in the first panel in Fig. 6(1a). In order to obtain a tree that explains $(u, v)$, we can modify $(T, \lambda)$ locally to obtain a tree $\left(T^{\prime}, \lambda^{\prime}\right)$ by inserting a single inner 1-edge $(a, b)$ in such a way that $b$ becomes the new root of $T^{*}$ and $u$ is adjacent to $a$ and $v$ adjacent to $b$ in $\left(T^{\prime}, \lambda^{\prime}\right)$. Thus, $u$ and $v$ are not siblings anymore. Moreover, we keep all edge labelings and set $\lambda^{\prime}(a, u)=\lambda^{\prime}(b, v)=0$. By construction, $\mathcal{X}[u, v]_{\left(T^{\prime}, \lambda^{\prime}\right)}=\{(u, v)\}$. We note that lca $(u, v)$ cannot be the root of $T$ since we have a triangle of the form $D$, i.e., there must be an inner 1-edge ancestral to lca $(u, v)$. One easily checks that $\left(T^{\prime}, \lambda^{\prime}\right)$ still explains all remaining pairs in $X \backslash X[u, v]$. Hence $\left(T^{\prime}, \lambda^{\prime}\right)$ explains $X$ whenever $X[u, v]=\{(u, v)\}$. It is least-resolved by construction and Lemma 11, and thus unique by Thm. 1 .

Analogously, a tree $\left(T^{\prime}, \lambda^{\prime}\right)$ that explains $\mathcal{X} \backslash X[u, v]$ with $X_{T, \lambda}[u, v]=(v, u)$ can be obtained from $(T, \lambda)$ by interchanging the roles of $u$ and $v$.

Finally, whenever $\left(T^{\prime}, \lambda^{\prime}\right)$ explains either $(u, v)$ or $(v, u)$ we can obtain a tree $(T, \lambda)$ that explains $u \mid v$ by "reversing" the contraction above. Because of the uniqueness of $\left(T^{\prime}, \lambda^{\prime}\right)$ it must locally look as in Fig. 6(1a) middle. That is, there is exactly one inner 1-edge along the path from $u$ to $v$ and all edges incident to $\operatorname{par}(v)$ must be 1-edges. Hence, after collapsing this edge to a single vertex, we obtain the least-resolved tree $(T, \lambda)$ that explains $u \mid v$. Since $\mathcal{X}_{\left(T^{\prime}, \lambda^{\prime}\right)}[u, z]=\mathcal{X}_{(T, \lambda)}[u, z]$ and $\mathcal{X}_{\left(T^{\prime}, \lambda^{\prime}\right)}[v, z]=\mathcal{X}_{(T, \lambda)}[v, z]$ is still true for all $z \in L\left(T^{\prime}\right)$, i.e., $\left(T^{\prime}, \lambda^{\prime}\right)$ explains $\mathcal{X}$ whenever $\mathcal{X}[u, v]=u \mid v$.

Case 1b. Suppose $\Delta_{u v}$ contains at least one triangle each of types $B, C$ and $D$, but not of type $A$. Then, $X[u, v] \in\{(u, v),[u, v]\}$. If $(T, \lambda)$ explains $[u, v]$ it has the following properties: Since $\Delta_{u v}$ contains triangles of type $B, v$ but not $u$ is a sink, and therefore $(\operatorname{par}(v), v)$ is a 1-edge while $(\operatorname{par}(u), u)$ is a 0-edge. Moreover, since $(v, u) \in X_{T, \lambda}$ and $(\operatorname{par}(u), u)$ is a 0 -edge, the path from lca $(u, v)$ to $u$ has to contain at least one 1-edge, $u$ and $v$ cannot have the same parent, hence $\operatorname{lca}(u, v) \succ \operatorname{par}(u)$. The presence of triangles of type $B, C$, and $D$ immediately implies that $(u, x) \in \mathcal{X}$ if and only if $(v, x) \in \mathcal{X}$ for all $x \in L(T) \backslash\{u, v\}$. Therefore, since each inner vertex of $(T, \lambda)$ (except possibly the root) must be connected to an outer 0-edge (Lemma 11(3b)), there cannot be any other inner vertex on the path from lca $(u, v)$ to $\operatorname{par}(u)$, hence the inner edge $(l c a(u, v), \operatorname{par}(u))$ must be present in $(T, \lambda)$. On the other hand, there must be a 0-edge $(\operatorname{par}(v), z)$ with $z \in L(T) \backslash\{u, v\}$ (Lemma 11(3b)). Moreover, $B, C$, and $D$ imply that there may be other 0 - or 1-edges incident to $\operatorname{par}(v)$. We denote by $T^{* *}$ the subtree rooted at $\operatorname{par}(u)$ that does not contain the leaf $u$. The subtree of $T$ that is rooted at $\operatorname{par}(v)$ but does neither contain the leaf $v$ nor the leaf $u$ nor any of the vertices of $T^{* *}$ is denoted by $T^{*}$. Thus, $(T, \lambda)$ must match the pattern shown Fig. $6(1 \mathrm{~b}$, left $)$. 
Case 1a

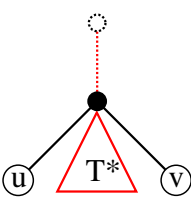

$u \mid v$

Case 3

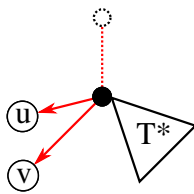

$[u, v] \in \mathcal{X}$

Case 4a

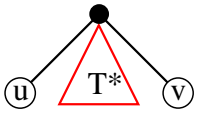

$u \mid v$

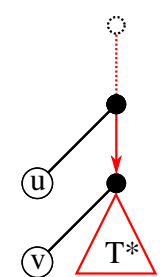

$(u, v) \in \mathcal{X}$

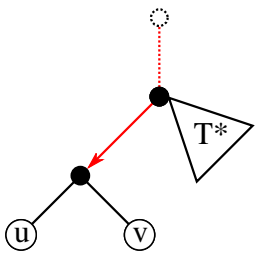

$u \mid v$

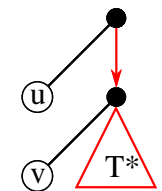

$(u, v) \in \mathcal{X}$

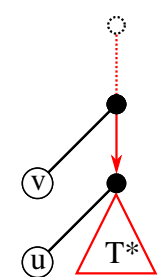

$(v, u) \in \mathcal{X}$
Case 1b

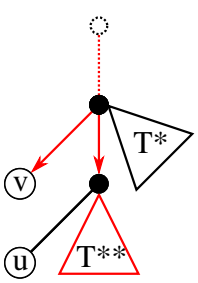

$[u, v] \in \mathcal{X}$

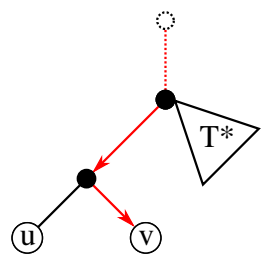

$(u, v) \in \mathcal{X}$

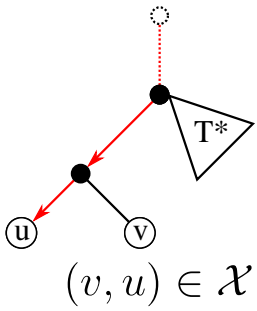

Case 4b

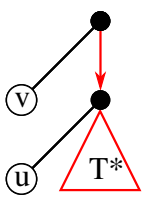

$(v, u) \in \mathcal{X}$

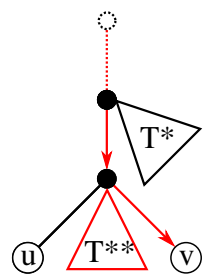

$(u, v) \in \mathcal{X}$

Figure 6: Local modifications of the tree $(T, \lambda)$ necessary to explain all possible choices of $X[u, v]$ for different combination of triangles types in $\Delta_{u v}$. Only the local environment around $u$ and $v$ is shown since the rest of the tree remains unchanged in all cases. Dashed lines indicate possible additional subtrees that are connected to the local situation by means of a 1-edge. The subtrees $T^{*}$ and $T^{* *}$ (with red triangles) may be attached to an inner vertex (via 1-edges); their internal structure is irrelevant for the arguments in the proof.

A tree $\left(T^{\prime}, \lambda^{\prime}\right)$ that explains $\mathcal{X}$ with $X[u, v]=(u, v)$ can be constructed by a simple change in the position of $v$ in $(T, \lambda)$, that is, we delete the 1-edge $(\operatorname{par}(v), v)$ and instead, insert the 1-edge $(\operatorname{par}(u), v)$. All other edge labels remain unchanged. By construction, $\mathcal{X}[u, v]_{\left(T^{\prime}, \lambda^{\prime}\right)}=\{(u, v)\}$ and again, one easily checks that $\left(T^{\prime}, \lambda^{\prime}\right)$ displays $X \backslash X[u, v]$ and therefore $X$. Moreover, $\left(T^{\prime}, \lambda^{\prime}\right)$ is by construction least-resolved and therefore uniquely defined. Hence, it must locally look as in Fig. $6(1 \mathrm{~b}$, right). Reverting the local modifications in $\left(T^{\prime}, \lambda^{\prime}\right)$ again yields the uniquely defined least-resolved tree $(T, \lambda)$ that explains $X$ with $X[u, v]=[u, v]$.

Case 2a. Suppose $\Delta_{u v}$ contains at least one triangle each of types $A$ and $C$ but no triangles of types $B$ and $D$. Then $X[u, v] \in\{u \mid v,(u, v),(v, u)\}$. We first assume that $(T, \lambda)$ explains $u \mid v$. Then, as in Case 1a, $u$ and $v$ have to be siblings, none of them is a sink and no other 0 -edge is incident to lca $(u, v)$. Hence, $(T, \lambda)$ locally looks again like Case 1a in Fig. 6. Local transformations of $(T, \lambda)$ that are completely analogous to Case la can be applied to $(T, \lambda)$ in order to obtain unique least-resolved trees that explain $X$ with $X[u, v]=(u, v)$ and $X[u, v]=(v, u)$, respectively (see Fig. 6(1a)). It is not hard to check that these transformations can be reversed by contraction of the edge $(v, u)$.

Case 2b. If $\Delta_{u v}$ contains at least one triangle each of types $A$ and $D$ but no triangles of types $B$ and $C$ then exactly the same arguments as in Cases $2 \mathrm{a}$ and $1 \mathrm{a}$ apply.

Case 2c. Suppose $\Delta_{u v}$ contains at least one triangle each of types $B$ and $C$ but no triangles of types $A$ or $D$. Then $\mathcal{X}[u, v] \in\{(u, v),[u, v]\}$. Let us assume that $(T, \lambda)$ explains $[u, v] \in \mathcal{X}$. As in Case $1 \mathrm{~b}$, the presence of triangles of type $B$ implies that $v$ but not $u$ is a sink. Arguing as in Case $1 \mathrm{~b}$ shows that $(T, \lambda)$ locally looks like Case $1 \mathrm{~b}$ in Fig. 6. The local transformation to the least-resolved tree $\left(T^{\prime}, \lambda^{\prime}\right)$ that explains $X$ with $X[u, v]=(u, v)$ can be performed as described in Case $1 \mathrm{~b}$, resulting in a tree $\left(T^{\prime}, \lambda^{\prime}\right)$ that locally looks like Fig. 6(1b). The same arguments as in Case $1 \mathrm{~b}$ can be applied to show that $\left(T^{\prime}, \lambda^{\prime}\right)$ explains $X$ and that there is a uniquely defined reverse transformation that converts $\left(T^{\prime}, \lambda^{\prime}\right)$ into $(T, \lambda)$.

Case 2d. If $\Delta_{u v}$ contains at least one triangle each of types $B$ and $D$ but no triangles of types $A$ and $C$ exactly the same arguments as in Cases $2 \mathrm{c}$ and $1 \mathrm{~b}$ apply.

Case 3. Suppose $\Delta_{u v}$ contains at least one triangle each of types $C$ and $D$ but no triangles of types $A$ and $B$. Then, $X[u, v] \in\{[u, v],(u, v),(v, u), u \mid v\}$. Assume that $(T, \lambda)$ explains $[u, v] \in X$. This implies that both $u$ and $v$ are sinks of $\mathcal{X}$, i.e., $(\operatorname{par}(u), u)$ and $(\operatorname{par}(v), v)$ are both 1-edges. By symmetry, $(u, x) \in \mathcal{X}$ if and only if $(v, x) \in \mathcal{X}$ and $(x, u) \in \mathcal{X}$ if and only if $(x, v) \in \mathcal{X}$, respectively, holds for all $x \in L(T) \backslash\{u, v\}$. 
We continue to show that $u$ and $v$ must be siblings. Assume for contradiction, they are not. Lemma 11(3) implies that there are leaves $z, z^{\prime} \in L(T) \backslash\{u, v\}$ such $(\operatorname{par}(u), z)$ and $\left(\operatorname{par}(v), z^{\prime}\right)$ are 0-edges. Hence, we have at least one of the cases $(u, z) \notin \mathcal{X}$ but $(v, z) \in \mathcal{X}$ or $\left(v, z^{\prime}\right) \notin X$ but $\left(u, z^{\prime}\right) \in \mathcal{X}$. If $\operatorname{par}(u)$ and $\operatorname{par}(v)$ are incomparable in $T$, even both cases are true. However, we obtain a contradiction to " $(v, x) \in \mathcal{X}$ iff $(u, x) \in X$ '. Thus, $u$ and $v$ are siblings. Since $(\operatorname{par}(u), u)$ and $(\operatorname{par}(v), v)$ are both 1-edges, there must be a leaf $y \in L(T) \backslash\{u, v\}$ such that the edge $(\operatorname{lca}(u, v), y)$ is a 0 -edge by Lemma 11(3). We denote by $T^{*}$ the subtree rooted at lca $(u, v)$ without the leaves $u$ and $v$.

Given $(T, \lambda)$, a tree $\left(T^{\prime}, \lambda^{\prime}\right)$ that displays $X$ with $X[u, v]=u \mid v$ is obtained by inserting an inner 1-edge $(a, b)$ such that $a$ becomes the new root of $T^{*}$ and $b=\operatorname{lca}_{T^{\prime}}(u, v)$. The outer edges $(b, u)$ and $(a, v)$ are 0 -edges; all other edge labels are retained as in $(T, \lambda)$. The resulting tree locally looks as illustrated in Fig. 6(Case 3). Relabeling of edges in $\left(T^{\prime}, \lambda^{\prime}\right)$ such that $(b, v)$ becomes a 1-edge yields the tree $\left(T^{\prime \prime}, \lambda^{\prime \prime}\right)$ that explains $X$ with $(u, v) \in X$. Similarly, converting the edge $(b, u)$ of $\left(T^{\prime}, \lambda^{\prime}\right)$ into a 1 -edge yields the tree $\left(T^{\prime \prime \prime}, \lambda^{\prime \prime \prime}\right)$ that explains $X$ with $(v, u) \in \mathcal{X}$. The trees $\left(T^{\prime}, \lambda^{\prime}\right),\left(T^{\prime \prime}, \lambda^{\prime \prime}\right)$, and $\left(T^{\prime \prime \prime}, \lambda^{\prime \prime \prime}\right)$ explain $X$ with the corresponding choice of $\mathcal{X}[u, v]$ and are least-resolved and thus unique. As in the previous cases, the reverse transformations are therefore also uniquely defined.

Case 4a. Suppose that all triangles in $\Delta_{u v}$ are of the form $A$. Then $X[u, v] \in\{u \mid v,(u, v),(v, u)\}$. Let us assume that $(T, \lambda)$ displays $u \mid v$. Then, $u$ and $v$ are both sources, hence $(\operatorname{par}(u), u)$ and $(\operatorname{par}(v), v)$ are both 0 -edges. Note that in contrast to Case $1 \mathrm{a}$, there is no $x \in L(T) \backslash\{u, v\}$ with $(x, u) \in \mathcal{X}$ or $(x, v) \in \mathcal{X}$. This implies that $u$ and $v$ are both incident to the root $\rho_{T}$ of $(T, \lambda)$ and among all edges incident to the root, $\left(\rho_{T}, u\right)$ and $\left(\rho_{T}, v\right)$ are the only 0 -edges. The tree $(T, \lambda)$ explaining $X[u, v]=u \mid v$ is shown Fig. $6(\mathrm{Case} 4 \mathrm{a})$. Note, the tree structure is very similar to Case 1a. Therefore, as in Case 1a, $(T, \lambda)$ can be locally modified to a least-resolved tree $\left(T^{\prime}, \lambda^{\prime}\right)$ explaining $X$ with $X[u, v]=(u, v)$ by introducing the single 1-edge $(a, b)$ with $a=\operatorname{par}(u), b=\operatorname{par}(v)$. The vertex $b$ becomes the root of $T^{*}$, where $T^{*}$ is defined as in Case 1a (see Fig. $6($ Case $4 \mathrm{a}))$. We set $\lambda^{\prime}(a, u)=0$ and $\lambda^{\prime}(b, v)=0$, while all other edge-labels are retained.

Exchanging the roles of $u$ and $v$ in $\left(T^{\prime}, \lambda^{\prime}\right)$ defines a least-resolved tree $\left(T^{\prime \prime}, \lambda^{\prime \prime}\right)$ that explains $X[u, v]=$ $(v, u)$. As in the previous cases, one easily verifies that all resulting trees are least-resolved and explain $X$ with the corresponding choice for $X[u, v]$. Hence the reverse transformations are also uniquely defined.

Case 4b. Suppose that $\Delta_{u v}$ contains only triangles of the form $B$. Hence, $X[u, v] \in\{(u, v),[u, v]\}$. Let us first assume that the least-resolved tree $(T, \lambda)$ explains $[u, v] \in X$. It immediately follows that $v$ is a sink and $u$ is not, hence $\lambda(\operatorname{par}(v), v)=1$ and $\lambda(\operatorname{par}(u), u)=0$. Moreover, we have $(v, u) \in X$, thus $\operatorname{par}(v) \succ$ $\operatorname{par}(u)$. Since for any $x \in L \backslash\{u, v\}$ it holds $(x, u) \notin X$ and thus, $\operatorname{par}(u) \succeq \operatorname{lca}(u, x)$, we have $\operatorname{par}(v)=\rho_{T}$ and $\operatorname{deg}\left(\rho_{T}\right)=2$. Therefore, $(T, \lambda)$ locally looks as in Fig. 6(Case $\left.4 \mathrm{~b}\right)$. Note that the tree structure is very similar to Case $1 \mathrm{~b}$. Hence, similar as in Case $1 \mathrm{~b},(T, \lambda)$ can be modified locally to a least-resolved tree $\left(T^{\prime}, \lambda^{\prime}\right)$ that displays $(u, v) \in X$ by contraction of $(\operatorname{par}(v), \operatorname{par}(u))$ and keeping all other edge-labels (see Fig. 6(Case 4b, right)). By the same argumentation as before, the reverse transformation is also uniquely defined.

Case 4c. Let us assume that all triangles in $\Delta_{u v}$ are of the form $C$, i.e., $X[u, v] \in\{[u, v], u \mid v,(u, v),(v, u)\}$, and that $(T, \lambda)$ explains $[u, v] \in \mathcal{X}$. As in Case 3, both $u$ and $v$ are sinks of $\mathcal{X}$, i.e., $(\operatorname{par}(u), u)$ and $(\operatorname{par}(v), v)$ are both 1-edges. Using the same symmetry argument as in Case 3, we conclude for any $x \in L(T) \backslash\{u, v\}$ that $(u, x) \in \mathcal{X}$ if and only if $(v, x) \in X$, and $(x, u) \in X$ if and only if $(x, v) \in X$, respectively. Following the arguments laid out in Case 3 , we conclude that $(T, \lambda)$ locally looks as Case 3 of Fig. 6 . Thus the local transformations described above can be applied analogously in order to obtain least-resolved trees that explain all possible $\mathcal{X}[u, v]$.

Case 4d. If $\Delta_{u v}$ contains only triangles of the form $D$, then we can apply the same construction as in Case $4 \mathrm{a}$ and 3 in order to conclude that $X$ can be explained for all possible $\mathcal{X}[u, v]$.

\section{Algorithmic Considerations}

Summarizing our results, we present two different algorithms that are both able to recognize a Fitch relation and compute its unique least-resolved tree. The first algorithm checks all induced triangles for forbidden subgraphs and, once recognized a Fitch relation, uses the set of informative triple as an input for the algorithm BUILD. Then, it simply labels the edges of the resulting Aho tree in the correct way. This is a very intuitive way to check for Fitch relations and construct the least-resolved tree, which we will make precise first. We shall see that it is possible, however, to achieve a much better performance by using that fact that Fitch graphs are di-cographs. One can alternatively check for Fitch relations using properties of di-cographs and build the least-resolved tree from the corresponding cotree. This can be achieved in linear time.

We have seen in the previous sections that every valid relations $X$ is explained by a unique, least-resolved tree $\left(T_{X}, \lambda_{X}\right)$, which, in turn, is identified by a set $r_{I}(X)$ of informative triples due to Lemma 12 . Lemma 1 


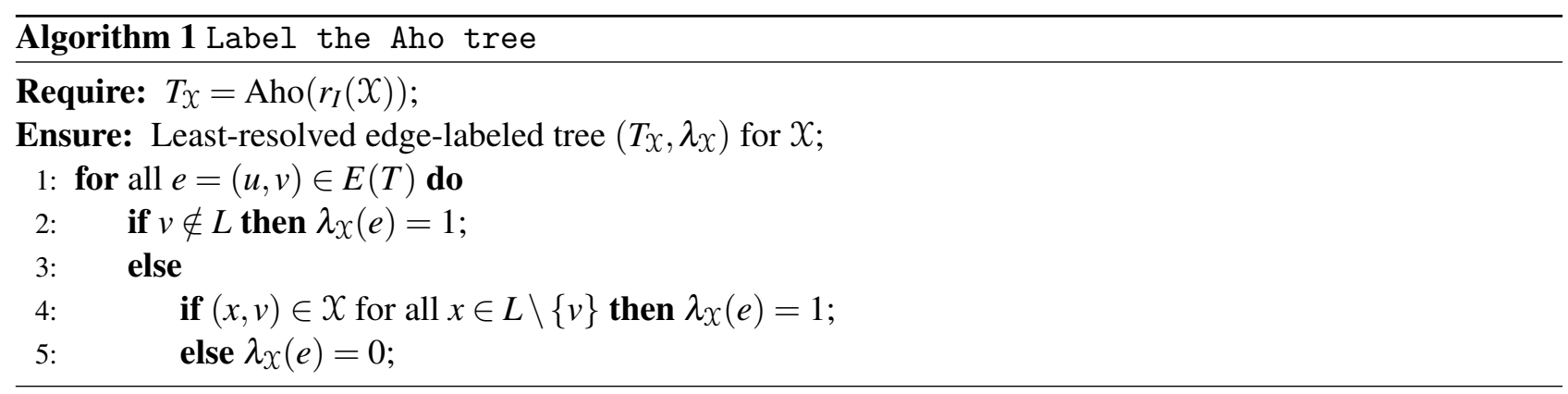

therefore implies

$$
T_{X}=\operatorname{Aho}\left(r_{I}(X)\right)
$$

It remains to construct the labeling function $\lambda_{X}$ on $\operatorname{Aho}\left(r_{I}(X)\right)$.

Lemma 16. Given the topology $T_{X}$ of the unique least-resolved tree explaining $X$, Algorithm 1 computes its correct unique edge labeling $\lambda_{x}$ in $\mathcal{O}(\max \{|X|,|L|\})$ time.

Proof. By Lemma 11 all inner edges $e$ of $\operatorname{Aho}\left(r_{I}(X)\right)$ must be labeled $\lambda(e)=1$ since otherwise they could be contracted, and hence, the tree would not be least-resolved. Now consider an edge $e=(u, v)$ leading to a leaf $v \in L$. If $(x, v) \notin X$ for some $x \in L \backslash\{v\}$ then $\lambda(e)=0$. Conversely, if $\lambda(e)=0$ then $(x, v) \notin X$ for every leaf below the siblings of $u$. At least one such leaf $x$ exists in a phylogenetic tree. Hence an outer edge is labeled $\lambda(e)=1$ if and only if $(x, v) \in X$ for all $x \in L \backslash\{v\}$.

For the time-complexity note that the labeling Algorithm 1 requires $\mathcal{O}\left(\mid E\left(T_{X} \mid\right)\right)$ operations to label the inner edges. To label the $|L|$ outer edges $(u, v)$ we have to determine the degree of vertex $v$ in $X$, that is, $\operatorname{deg}(v)=1$ implies that $(u, v)$ is an outer edge, which requires $\mathcal{O}(\max \{|\mathcal{X}|,|L|\})$ operations. Since $\left|E\left(T_{X}\right)\right|$ is bounded by $\mathcal{O}(|L|)$, the total running time of the labeling step is bounded by $\mathcal{O}(\max \{|\mathcal{X}|,|L|\})$.

A tree explaining a given Fitch relation can be obtained by the following procedure: First, we check whether $X$ is a Fitch relation. This can be achieved in $\mathcal{O}\left(|L|^{3}\right)$ by checking validity of the $\left(\begin{array}{c}L \\ 3\end{array}\right)$ induced triangles. If $X \subset L \times L$ is a Fitch relation, then $r_{I}(X)$ can be constructed within $\mathcal{O}\left(|L|^{3}\right)$ time. For a given the set of triples $R=r_{I}(X)$, the original approach to check whether $R$ is consistent (in which case $\operatorname{Aho}(R)$ is returned) or not, has time complexity $\mathcal{O}(|R||L|)$ [1]. However, various further practical implementations have been described $[10,25,27,28]$ that improve the asymptotic performance. Constructing $\operatorname{Aho}(R)$ and using Algorithm 1 to obtain the edge labels, it is therefore possible to recognize a Fitch relation $\mathcal{X}$ and to compute its respective (least-resolved) tree $(T, \lambda)$ in $\mathcal{O}\left(|L|^{4}\right)$.

It is possible to improve the algorithms to recognize Fitch relations $\mathcal{X}$ and compute its least-resolved tree $(T, \lambda)$ in the following way: Every di-cograph $G$ is explained by a unique cotree $\left(T^{\prime}, t\right)[35,36]$, that is, an ordered phylogenetic tree $T^{\prime}$ with leaf set $V(G)$ and a vertex-labeling function $t: V^{0}\left(T^{\prime}\right) \rightarrow\{0,1, \overrightarrow{1}\}$, such that $t(u) \neq t(v)$ for all inner edges $(u, v)$ in $T^{\prime}$, defined by

$$
t(\operatorname{lca}(x, y))= \begin{cases}0, & \text { if }(x, y)(y, x) \notin E(G) \\ 1, & \text { if }(x, y)(y, x) \in E(G) \\ \overrightarrow{1}, & \text { else } .\end{cases}
$$

Since the vertices in the cotree $T^{\prime}$ are ordered, the label $\overrightarrow{1}$ on some lca $(x, y)$ of two distinct leaves $x, y \in L$ means that there is an edge $(x, y) \in E(G)$, while $(y, x) \notin E(G)$, whenever $x$ is placed to the left of $y$ in $T^{\prime}$ [22]. As discussed in Section 3, any di-cograph that does not contain the invalid triangles $F_{1}, F_{5}$ and $F_{8}$ is a Fitch graph.

Lemma 17. Let $G$ be a di-cograph and $\left(T^{\prime}, t\right)$ its corresponding cotree. A di-cograph contains the triangle $F_{1}, F_{5}$ and $F_{8}$ as an induced subgraph if and only if there are two vertices $v, w \in V^{0}\left(T^{\prime}\right)$ with $v \succ_{T^{\prime}} w$ such that either $(i) t(v)=0 \neq t(w)$ or $(i i) t(v)=\overrightarrow{1}, t(w)=1$ and $w$ is located in some subtree (rooted at a child of $v)$ that is different from the subtree rooted at the right-most child of $v$.

Proof. Consider first the triangles $F_{1}$ and $F_{5}$ with vertices $x, y, z$ and edge set $E\left(F_{1}\right)=\{(x, y)\}$ and $E\left(F_{5}\right)=$ $\{(x, y),(y, x)\}$. Equivalently, we have $t\left(\operatorname{lca}_{T^{\prime}}(x, y)\right) \in\{1, \overrightarrow{1}\}, t\left(\operatorname{lca}_{T^{\prime}}(x, y, z)\right)=0$ and $v=\operatorname{lca}_{T^{\prime}}(x, y, z) \succ_{T^{\prime}}$ $w=\operatorname{lca}_{T^{\prime}}(x, y)$.

Now, let $F_{8}$ have vertices $x, y, z$ and edge set $E\left(F_{8}\right)=\{(x, y),(y, x),(x, z),(y, z)\}$. Equivalently, we have $t\left(\operatorname{lca}_{T^{\prime}}(x, y)\right)=1, t\left(\operatorname{lca}_{T^{\prime}}(x, y, z)\right)=\overrightarrow{1}$ and $v=\operatorname{lca}_{T^{\prime}}(x, y, z) \succ_{T} w=\operatorname{lca}_{T^{\prime}}(x, y)$. In particular, $x$ and $y$ must 

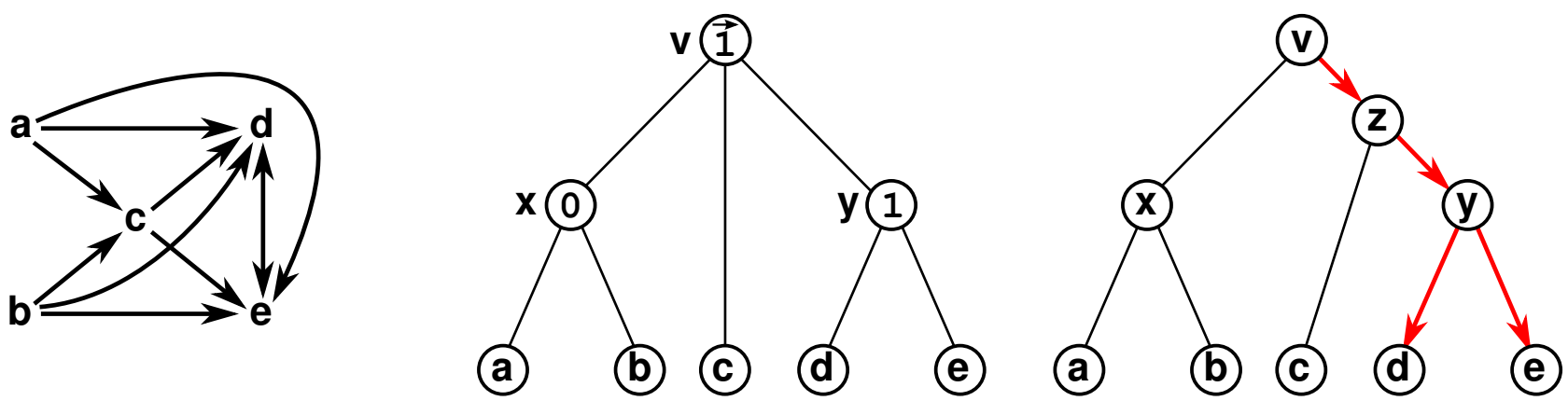

Figure 7: Application of cotree2fitchtree: A Fitch-relation $X$ (left), its cotree $\left(T^{\prime}, t\right)$ (middle) and a tree $(T, \lambda)$ that explains $\mathcal{X}$ (right) is shown. The tree $(T, \lambda)$ is obtained from $\left(T^{\prime}, t\right)$ by replacing the subtree with vertices $v, x, y$ and $c$ by the caterpillar $(x(c, y))$ rooted at $v$ and adding the edge-labels as described in the procedure cotree2fitchtree. By Lemma 17, $t(x)=0$ for all inner vertices $x$ in the subtrees left from the subtree rooted a the right-most child $y$ of $v$. Note, $(T, \lambda)$ is not least-resolved w.r.t. $X$. Nevertheless, Theorem 1 implies that $(T, \lambda)$ is displayed by the least-resolved tree for $X$. Here, the least-resolved tree can be obtained from $(T, \lambda)$ by contracting the edges $(v, x)$ and $(z, y)$.

be placed left from $z$ in $T^{\prime}$ and therefore, $w$ must be located in some subtree different from the subtree rooted at the right-most child of $v=\operatorname{lca}_{T}(x, y, z)$.

Corollary 1. Let $X$ be a Fitch graph and $\left(T^{\prime}, t\right)$ its corresponding cotree. If $X$ contains an edge, then it is weakly connected, i.e., the underlying undirected graph obtained from $\mathcal{X}$ by ignoring the direction of the edges is connected. Moreover, any vertex $x \prec v$ for which $t(v)=0$ must be a leaf of $T^{\prime}$.

Proof. If a Fitch graph $X$ contains an edge, then its cotree contains an inner vertex labeled 1 or $\overrightarrow{1}$. If $X$ is disconnected, then the root of the cotree must be labeled 0 and Lemma 17 implies that $\mathcal{X}$ is not a Fitch graph. Thus, the root must be labeled either 1 or $\overrightarrow{1}$, which implies that $X$ is weakly connected.

Now assume that $\left(T^{\prime}, t\right)$ contains a vertex $v$ with $t(v)=0$. Let $x \prec v$ with $(v, x) \in E\left(T^{\prime}\right)$ and assume for contradiction that $x$ is an inner vertex. By the definition of cotrees, $t(v)=0 \neq t(x)$. Lemma 17 and Theorem 2 imply that $X$ is not a Fitch graph; a contradiction.

Verifying whether a graph $G$ is a di-cograph or not can be achieved in $\mathcal{O}(|V(G)|+|E(G)|)$ time, see $[22,35]$ for further details. To verify that a given di-cograph $G$ does not contain $F_{1}, F_{5}$ and $F_{8}$ as an induced subgraph, we apply the classical Breadth-first search (BFS) [7] on its cotree $\left(T^{\prime}, t\right)$ starting with the root and check whether there are invalid combinations of vertex labels in $\left(T^{\prime}, t\right)$ according to Lemma 17. Note, $L\left(T^{\prime}\right)=V(G)$ and $\left|V^{0}\left(T^{\prime}\right)\right| \leq\left|L\left(T^{\prime}\right)\right|-1$. Thus, the BFS-method runs in $\mathcal{O}\left(\left|V\left(T^{\prime}\right)\right|\right)=\mathcal{O}(|V(G)|)$ time. Therefore, recognition of Fitch graphs, or equivalently, Fitch relations can be achieved within $\mathcal{O}(|V(G)|+$ $|E(G)|)$ time.

We show now how to obtain a tree $(T, \lambda)$ that explains a Fitch relation $\mathcal{X}$ from its cotree representation $\left(T^{\prime}, t\right)$. To this end we need to translate the (ordered) cotree with vertex labels " 0 ", " 1 " and " $\overrightarrow{1}$ " to an unordered tree with edge labels " 1 " and " 0 ", summarized next and called cotree2fitchtree:

For all $x \in V^{0}\left(T^{\prime}\right)$, if

$t(x)=1$ (resp. 0), then set for each child $y$ of $x$ the label $\lambda(x, y)=1$ (resp. 0), and else,

$t(x)=\overrightarrow{1}$, then we can assume w.l.o.g. that the children of $x$ are ordered $x_{1}, \ldots, x_{k}, k \geq 2$ from left to right. Now, replace the subtree of $T^{\prime}$ with vertices $x$ and $x_{1}, \ldots, x_{k}$ by the caterpillar $C\left(x_{1}, \ldots, x_{k}\right):=$ $\left(x_{1}\left(x_{2}\left(\ldots\left(x_{k-1}, x_{k}\right) \ldots\right)\right.\right.$ (in Newick notation) that is rooted at $x$. Set the label $\lambda$ of all inner edges of $C\left(x_{1}, \ldots, x_{k}\right)$ and the outer-edge incident to $x_{k}$ to " 1 " and the labels of all other (outer) edges of $C\left(x_{1}, \ldots, x_{k}\right)$ to " 0 ". Note that outer edges of $C\left(x_{1}, \ldots, x_{k}\right)$ may be inner edges in $(T, \lambda)$.

Finally, remove all vertex labels and ignore the ordering of the vertices to obtain the tree $(T, \lambda)$.

For an example of cotree2fitchtree see Figure 7.

Lemma 18. The procedure cotree2fitchtree transforms the cotree $\left(T^{\prime}, t\right)$ of a Fitch-relation $X$ into a tree $(T, \lambda)$ that explains $X$ in $\mathcal{O}\left(\left|V\left(T^{\prime}\right)\right|\right)$ time. 
Proof. Let $\left(T^{\prime}, t\right)$ be the cotree of the Fitch-relation $X$ and $(T, \lambda)$ the tree resulting from cotree2fitchtree. Since all inner vertices of $\left(T^{\prime}, t\right)$ are labeled, each edge of $(T, \lambda)$ receives a label " 0 " or " 1 " by construction. It needs to be verified that $(T, \lambda)$ explains $X$.

Assume that $(x, y),(y, x) \in X$. Hence, $t\left(\operatorname{lca}_{T^{\prime}}(x, y)\right)=1$. By construction, the edges incident to the children of $v=\operatorname{lca}_{T^{\prime}}(x, y)$ are labeled " 1 ". Hence, both paths in $(T, \lambda)$ from $\operatorname{lca}_{T}(x, y)=v$ to $x$ and to $y$ contain 1-edges. Thus, $(T, \lambda)$ explains all symmetric pairs in $X$.

Assume that $(x, y),(y, x) \notin X$ and let $z=\operatorname{lca}_{T^{\prime}}(x, y)$. Hence, $t(z)=0$. Cor. 1 implies that $(z, x)$ and $(z, y)$ are outer edges in $T^{\prime}$ that are, by construction, labeled " 0 " in $(T, \lambda)$. As a consequence, the path from $x$ to $y$ in $(T, \lambda)$ contains only 0 -edges, which implies that $(T, \lambda)$ also explains that all pairs $(x, y),(y, x)$ that are not contained in $X$.

Assume $(x, y) \in \mathcal{X}$ and $(y, x) \notin X$. Hence, $t\left(\operatorname{lca}_{T^{\prime}}(x, y)\right)=\overrightarrow{1}$ and $x$ is left from $y$ in $T^{\prime}$. Let $v_{i}$ and $v_{j}$ be children of $\operatorname{lca}_{T^{\prime}}(x, y)$ with $v_{i} \succeq x$ and $v_{j} \succeq y$. Since $x$ is left from $y$, also $v_{i}$ is left from $v_{j}$ in $T^{\prime}$. Note, $v_{i}$ and $v_{j}$ are now part of the inserted caterpillar $C\left(\operatorname{child}\left(\operatorname{lca}_{T^{\prime}}(x, y)\right)\right)$ in $(T, \lambda)$. Therefore, $\operatorname{lca}_{T}(x, y)$ must be an inner vertex of this caterpillar. By construction, the path from $\operatorname{lca}_{T}(x, y)$ to $v_{j} \succeq y$ contains a 1-edge and thus $(x, y) \in X$. It remains to show that the path from $\operatorname{lca}_{T}(x, y)$ to $x$ contains only 0 -edges so that $(y, x) \notin X$. Note that the vertex $v_{i}$ is a child of $\operatorname{lca}_{T}(x, y)$ in $T$ and the edge $\left(\operatorname{lca}_{T}(x, y), v_{i}\right)$ is labeled " 0 ". Thus, if $v_{i}=x$ we are done. Assume that $v_{i} \neq x$ and hence, that $v_{i}$ is an inner vertex of $T^{\prime}$. By the definition of cotrees, we have $t\left(\operatorname{lca}_{T^{\prime}}(x, y)\right)=\overrightarrow{1} \neq t\left(v_{i}\right)$. Since $v_{i}$ is left from $v_{j}$ in $T^{\prime}$ we can apply Lemma 17 and conclude that $t\left(v_{i}\right) \neq 1$. Hence, there is only one possibility left, namely $t\left(v_{i}\right)=0$. Cor. 1 implies that $\left(v_{i}, x\right)$ must must be an outer edge in $\left(T^{\prime}, t\right)$ that - by construction - is labeled " 0 " in $(T, \lambda)$. Hence, the path from lca $(x, y)$ to $x$ contains only 0 -edges and therefore, $(y, x) \notin X$.

For the running time, observe that the edge-label in each step of cotree 2 itchtree for vertices $v$ with $t(v) \in\{0,1\}$ can be computed in $\mathcal{O}\left(\operatorname{deg}_{T^{\prime}}(v)\right)$ time. Moreover, if $t(v)=\overrightarrow{1}$ for some vertex $v$ in $\left(T^{\prime}, t\right)$, we have to replace the subtree induced by $v$ and its children $v_{1}, \ldots, v_{k}$ (ordered from left to right) in $\left(T^{\prime}, t\right)$, by the edge-labeled caterpillar $C\left(v_{1}, \ldots, v_{k}\right)$. This task can also be performed in $\mathcal{O}\left(\operatorname{deg}_{T^{\prime}}(v)\right)$ time. Since each step in cotree2fitchtree can be done in $\mathcal{O}\left(\operatorname{deg}_{T^{\prime}}(v)\right)$ time and $\left.\sum_{v \in V^{0}\left(T^{\prime}\right)} \operatorname{deg}_{T^{\prime}}(v)\right) \leq 2\left|E\left(T^{\prime}\right)\right|<2\left|V\left(T^{\prime}\right)\right|$, this implies a total time requirement of $\mathcal{O}\left(\left|V\left(T^{\prime}\right)\right|\right)$.

Let $(T, \lambda)$ be the tree that explains $X$ as constructed with cotree $2 \mathrm{f}$ itchtree from the respective cotree $\left(T^{\prime}, t\right)$. Theorem 1 implies that $(T, \lambda)$ displays the least-resolved tree for $X$. Thus, we can utilize Lemma 11 and contract all irrelevant edges and all inner 0-edges in $(T, \lambda)$ in order to obtain the least-resolved tree for $X$. The latter can be done in $\mathcal{O}(|V(T)|)$ time. Taking the latter results together with the observation that $|V(T)| \geq\left|V\left(T^{\prime}\right)\right|$, we obtain the following

Theorem 3. Verifying whether an irreflexive relation $X \subseteq L \times L$ is a Fitch relation or not, can be a achieved in $\mathcal{O}(|L|+|X|)$ time. Its unique least-resolved edge-labeled tree $\left(T_{X}, \lambda_{X}\right)$ can be computed in $\mathcal{O}\left(\left|V\left(T_{X}\right)\right|\right)=$ $\mathcal{O}(|L|)$ time, given the cotree of $X$.

The fact the Fitch graphs form a heritable family (cf. Lemma 3) has far-reaching consequences for computational problems such as the following:

Problem 1 (Fitch Graph Modification).

Given: $\quad$ a graph $G=(L, F)$ and integers $i, j, k$.

Question: Are there subsets $L^{\prime} \subseteq L, F^{\prime} \subseteq F$ and $F^{\prime \prime} \subseteq(L \times L) \backslash F$

with $\left|L^{\prime}\right| \leq i,\left|F^{\prime}\right| \leq j$ and $\left|F^{\prime \prime}\right| \leq k$ such that

$G-L^{\prime}-\bar{F}^{\prime}+F^{\prime \prime}$ is a Fitch graph?

A very general result on graph editing on heritary graph classes $[6,18]$ immediately implies

Corollary 2. Fitch Graph Modification is NP-complete, but fixed-parameter tractable and can be solved in $\mathcal{O}\left(3^{i+2 j+2 k}|L|^{4}\right)$ time.

\section{Concluding Remarks}

The relationships and mutual constraints of gene trees and species trees are by no means completely understood. Here we have attempted to identify the phylogenetic information that is contained in horizontal transfer events. An alternative approach to understand such relations has been explored by [22]. The relations considered there, however, are completely defined by the labeling of the inner gene tree vertices as speciation, duplication or HGT.

A more commonly used definition of xenology was proposed by Walter Fitch [15]. We formalized Fitch's concept of xenology in the form of a not necessarily symmetric binary relation $\mathcal{X}$ so that $(x, y) \in$ 
$X$ if and only the lineage from $\operatorname{lca}(x, y)$ to $y$ was horizontally transferred at least once. Our main result is a complete characterization of such relations in terms of forbidden induced subgraphs and a complete characterization of the minimally resolved trees explaining such relations. These Fitch trees represent the complete information on the gene tree that is "recorded" by the horizontal transfer events alone. Polynomialtime algorithms have been devised to compute Fitch trees from Fitch relations.

The practical usefulness of the Fitch relation and its trees eventually will depend on how easy or difficult it will turn out to estimate the Fitch relation from data. Although no convenient tools are available to our knowledge to identify directed xenology relationships without first reconstructing gene and species trees, this seems to be not at all a hopeless task, since genes that are imported by HGT from an ancestor of species $A$ into an ancestor of species $B$ are expected to be more closely related than expected from the bulk of the genome [40, 42]. Inference from real-life data will never be noise free. It is therefore encouraging that the corresponding editing problem is at least FPT even though it is NP complete as so many other computational problems in phylogenetics.

An interesting facet of the results is that the Fitch graphs are a proper subset of the di-cographs that naturally appear in a formalization of xenology that focuses on the vertices of the gene tree [22]. While our results strongly suggest that there should be a close relationship between these two models, it remains an open question what exactly this connection and its biological interpretation might be. A related question concerns the symmetrized version of the xenology relation: what can be said about the relation $X^{\text {sym }}$ with $\{x, y\} \in X^{\text {sym }}$ whenever $(x, y) \in X$, that is, $\{x, y\} \in X^{\text {sym }}$ iff there is a HGT event along the unique path from $x$ to $y$ in the gene tree? What if we knew that there is exactly one transfer event along the path?

\section{Acknowledgements}

We thank Maribel Hernández Rosales and her team for stimulating discussions. This work was funded in part by the BMBF-funded project "Center for RNA-Bioinformatics" (031A538A, de.NBI-RBC) and a travel grant from DAAD PROALMEX (Proj. No. 278966).

\section{References}

[1] A. V. Aho, Y. Sagiv, T. G. Szymanski, and J. D. Ullman. Inferring a tree from lowest common ancestors with an application to the optimization of relational expressions. SIAM Journal on Computing, 10(3):405-421, 1981

[2] A. M. Altenhoff, B. Boeckmann, S. Capella-Gutierrez, D. A. Dalquen, T. DeLuca, K. Forslund, H.C. Jaime, B. Linard, C. Pereira, L. P. Pryszcz, F. Schreiber, A. S. da Silva, D. Szklarczyk, C.-M. Train, P. Bork, O. Lecompte, C. von Mering, I. Xenarios, K. Sjölander, L. J. Jensen, M. J. Martin, M. Muffato, T. Gabaldón, S. E. Lewis, P. D. Thomas, E. Sonnhammer, and C. Dessimoz. Standardized benchmarking in the quest for orthologs. Nature Methods, 13:425-430, 2016.

[3] S. Böcker and A. W. M. Dress. Recovering symbolically dated, rooted trees from symbolic ultrametrics. Adv. Math., 138:105-125, 1998.

[4] D. Bryant. Building trees, hunting for trees, and comparing trees: theory and methods in phylogenetic analysis. PhD thesis, University of Canterbury, 1997.

[5] D. Bryant and M. Steel. Extension Operations on Sets of Leaf-Labeled Trees. Advances in Applied Mathematics, 16(4):425-453, Dec. 1995.

[6] L. Cai. Fixed-parameter tractability of graph modification problems for hereditary properties. Information Processing Letters, 58(4):171 - 176, 1996.

[7] T. H. Cormen, C. E. Stein, Clifford and Leiserson, and . R. L. Rivest. Introduction to algorithms. MIT press, Cambridge, Massachusetts, 2009.

[8] C. Crespelle and C. Paul. Fully dynamic recognition algorithm and certificate for directed cographs. Discr. Appl. Math., 154:1722-1741, 2006.

[9] M. C. H. Dekker. Reconstruction methods for derivation trees. Master's thesis, Vrije Universiteit, Amsterdam, Netherlands, 1986. 
[10] Y. Deng and D. Fernández-Baca. Fast Compatibility Testing for Rooted Phylogenetic Trees. In R. Grossi and M. Lewenstein, editors, 27th Annual Symposium on Combinatorial Pattern Matching (CPM 2016), volume 54 of Leibniz International Proceedings in Informatics (LIPICs), pages 12:112:12, Dagstuhl, Germany, 2016. Schloss Dagstuhl-Leibniz-Zentrum fuer Informatik.

[11] R. Dondi, N. El-Mabrouk, and M. Lafond. Correction of weighted orthology and paralogy relationscomplexity and algorithmic results. In International Workshop on Algorithms in Bioinformatics, pages 121-136. Springer, 2016.

[12] R. Dondi, G. Mauri, and I. Zoppis. Orthology correction for gene tree reconstruction: Theoretical and experimental results. Procedia Computer Science, 108:1115 - 1124, 2017. International Conference on Computational Science, ICCS 2017, 12-14 June 2017, Zurich, Switzerland.

[13] A. Ehrenfeucht and G. Rozenberg. Primitivity is hereditary for 2-structures. Theor. Comp. Sci., 70:343-359, 1990.

[14] W. M. Fitch. Distinguishing Homologous from Analogous Proteins. Systematic Biology, 19(2):99113, June 1970.

[15] W. M. Fitch. Homology a personal view on some of the problems. Trends Genet., 16(5):227-231, 2000.

[16] T. Gabaldon and E. V. Koonin. Functional and evolutionary implications of gene orthology. Nat. Rev. Genet., 14:360-366, 2013.

[17] S. Grünewald, M. Steel, and M. S. Swenson. Closure operations in phylogenetics. Mathematical Biosciences, 208(2):521-537, Aug. 2007.

[18] F. Gurski, R. I., J. Rothe, and E. Wanke. Exakte Algorithmen für schwere Graphenprobleme. SpringerVerlag, Berlin Heidelberg, 2010.

[19] M. Hellmuth. Biologically feasible gene trees, reconciliation maps and informative triples. Algorithms for Molecular Biology, 12(1):23, 2017.

[20] M. Hellmuth, M. Hernandez-Rosales, K. T. Huber, V. Moulton, P. F. Stadler, and N. Wieseke. Orthology relations, symbolic ultrametrics, and cographs. J. Math. Biology, 66(1-2):399-420, 2013.

[21] M. Hellmuth and C. Seemann. The matroid structure of representative triple sets and triple closure computation. arXiv:1707.01667, 2017. submitted.

[22] M. Hellmuth, P. F. Stadler, and N. Wieseke. The mathematics of xenology: Di-cographs, symbolic ultrametrics, 2-structures and tree-representable systems of binary relations. J. Math. Biol., 75(1):199237, 2017.

[23] M. Hellmuth and N. Wieseke. From sequence data including orthologs, paralogs, and xenologs to gene and species trees. In P. Pontarotti, editor, Evolutionary Biology: Convergent Evolution, Evolution of Complex Traits, Concepts and Methods, pages 373-392, Cham, 2016. Springer.

[24] M. Hellmuth, N. Wieseke, M. Lechner, H.-P. Lenhof, M. Middendorf, and P. F. Stadler. Phylogenomics with Paralogs. Proceedings of the National Academy of Sciences, 112(7):2058-2063, 2015.

[25] M. R. Henzinger, V. King, and T. Warnow. Constructing a Tree from Homeomorphic Subtrees, with Applications to Computational Evolutionary Biology. Algorithmica, 24(1):1-13, May 1999.

[26] M. Hernandez-Rosales, M. Hellmuth, N. Wieseke, K. T. Huber, and P. F. Moulton, V.and Stadler. From event-labeled gene trees to species trees. BMC Bioinformatics, 13(19):S6, 2012.

[27] J. Holm, K. de Lichtenberg, and M. Thorup. Poly-logarithmic Deterministic Fully-dynamic Algorithms for Connectivity, Minimum Spanning Tree, 2-edge, and Biconnectivity. J. ACM, 48(4):723760 , July 2001.

[28] J. Jansson, J. H.-K. Ng, K. Sadakane, and W.-K. Sung. Rooted Maximum Agreement Supertrees. Algorithmica, 43(4):293-307, Dec. 2005. 
[29] R. A. Jensen. Orthologs and paralogs - we need to get it right. Genome Biology, 2:interactions1002, Aug. 2001.

[30] E. V. Koonin. Orthologs, Paralogs, and Evolutionary Genomics. Annual Review of Genetics, 39(1):309-338, 2005

[31] M. Lafond, R. Dondi, and N. El-Mabrouk. The link between orthology relations and gene trees: A correction perspective. Algorithms for Molecular Biology, 11:4, Apr. 2016.

[32] M. Lafond and N. El-Mabrouk. Orthology and paralogy constraints: Satisfiability and consistency. BMC Genomics, 15(6):S12, Oct. 2014.

[33] M. Lafond and N. El-Mabrouk. Orthology relation and gene tree correction: complexity results. In International Workshop on Algorithms in Bioinformatics, pages 66-79. Springer, 2015.

[34] M. Lafond, M. Semeria, K. M. Swenson, E. Tannier, and N. El-Mabrouk. Gene tree correction guided by orthology. BMC Bioinformatics, 14(15):S5, Oct. 2013.

[35] R. M. McConnell and F. de Montgolfier. Linear-time modular decomposition of directed graphs. Discrete Applied Mathematics, 145(2):198-209, 2005.

[36] R. H. Möhring and F. J. Radermacher. Substitution decomposition for discrete structures and connections with combinatorial optimization. Ann. Discrete Math., 19:257-356, 1984.

[37] B. T. L. Nichio, J. N. Marchaukoski, and R. T. Raitzz. New tools in orthology analysis: A brief review of promising perspectives. Front Genet., 8:165, 2017.

[38] N. Nøjgaard, N. El-Mabrouk, D. Merkle, N. Wieseke, and M. Hellmuth. Partial orthology, paralogy and xenology relations - satisfiability in terms of di-cographs. arXiv:1711.00504v1, 2017.

[39] N. Nojgaard, M. Geiß, D. Merkle, P. F. Stadler, N. Wieseke, and M. Hellmuth. Forbidden Time Travel: Characterization of Time-Consistent Tree Reconciliation Maps. In R. Schwartz and K. Reinert, editors, 17th International Workshop on Algorithms in Bioinformatics (WABI 2017), volume 88 of Leibniz International Proceedings in Informatics (LIPICs), pages 17:1-17:12, Dagstuhl, Germany, 2017. Schloss Dagstuhl-Leibniz-Zentrum fuer Informatik.

[40] P. S. Novichkov, M. V. Omelchenko, M. S. Gelfand, A. A. Mironov, Y. I. Wolf, and E. V. Koonin. Genome-wide molecular clock and horizontal gene transfer in bacterial evolution. J. Bacteriology, 186:6575-6585, 2004.

[41] C. Rancurel, L. Legrand, and E. G. J. Danchin. Alienness: Rapid detection of candidate horizontal gene transfers across the tree of life. Genes, 8:E248, 2017.

[42] M. Ravenhall, N. Škunca, F. Lassalle, and C. Dessimoz. Inferring horizontal gene transfer. PLoS Comput Biol, 11:e1004095, 2015.

[43] C. Semple and M. Steel. Phylogenetics, volume 24 of Oxford Lecture Series in Mathematics and its Applications. Oxford University Press, Oxford, Feb. 2003.

[44] M. Steel. Phylogeny: Discrete and Random Processes in Evolution. CBMS-NSF Regional Conference Series in Applied Mathematics. Society for Industrial and Applied Mathematics, Philadelphia, Nov. 2016. 